\title{
Twisted complexes on a ringed space as a dg-enhancement of the derived category of perfect complexes
}

\section{Zhaoting Wei ${ }^{1}$}

\begin{abstract}
We study the dg-category of twisted complexes on a ringed space and prove that it gives a new dg-enhancement of the derived category of perfect complexes on that space. A twisted complex is a collection of locally defined sheaves together with the homotopic gluing data. We construct a dg-functor from twisted complexes to perfect complexes, which turns out to be a dg-enhancement. This new enhancement has the advantage of being completely geometric and it comes directly from the definition of perfect complex. In addition we will talk about some applications and further topics around twisted complexes.
\end{abstract}

Keywords Twisted complex $\cdot$ Dg-enhancement $\cdot$ Perfect complex $\cdot$ Higher structure

Mathematics Subject Classification 14F05 - 16E45 · 18E30

\section{Introduction}

The derived categories of perfect complexes and pseudo-coherent complexes on ringed topoi were introduced in SGA 6 [2]. They have played an important role in mathematics ever since. Nevertheless we would like to consider the differential graded (dg)-enhancements of these derived categories. More precisely we have the following definition.

Definition 1.1 Let $\mathcal{C}$ be a triangulated category. A dg-enhancement of $\mathcal{C}$ is a pair $(\mathcal{B}, \varepsilon)$ where $\mathcal{B}$ is a pre-triangulated dg-category and

Zhaoting Wei

zhaotwei@indiana.edu

1 Department of Mathematics, Indiana University, 831 E 3rd St, Bloomington, IN 47405, USA 


$$
\varepsilon: \mathrm{HoB} \stackrel{\sim}{\longrightarrow} \mathrm{C}
$$

is an equivalence of triangulated categories. Here HoB is the homotopy category of B.

For the derived category $\mathrm{D}_{\text {perf }}(X)$ on a (quasi-compact and separated) scheme $X$ we have the classical injective enhancement, which consists of h-injective objects, see [12, Section 3.1]. Although very useful, the injective resolution has its drawback that the modules are too "large" and the construction is not geometric. Therefore we are seeking for a new, more geometric dg-enhancement.

In the late 1970's Toledo and Tong [19] introduced twisted complexes as a way to get their hands on perfect complexes of sheaves on a complex manifold and implicitly they recognized this was a dg-model for the derived category of perfect complexes. In this paper we prove in all details that twisted complexes form a dg-model for categories of perfect complexes (and more generally pseudo-coherent complexes) of sheaves on a ringed space under some conditions.

Let us first give an informal description to illustrate the idea of twisted complexes. Recall that a complex of sheaves $\mathcal{S}^{\bullet}$ on $X$ is perfect if for any point $x \in X$, there exist an open neighborhood $x \in U \subset X$ and a two-side bounded complex of finitely generated locally free sheaves $E_{U}^{\bullet}$ on $U$ together with a quasi-isomorphism

$$
\theta_{U}:\left.E_{U}^{\bullet} \stackrel{\sim}{\longrightarrow} \mathcal{S}^{\bullet}\right|_{U}
$$

For two different open subsets $U_{i}$ and $U_{j}$ we have two quasi-isomorphisms

$$
\theta_{i}:\left.E_{U_{i}}^{\bullet} \stackrel{\sim}{\longrightarrow} \mathcal{S}^{\bullet}\right|_{U_{i}} \quad \text { and } \quad \theta_{j}:\left.E_{U_{j}}^{\bullet} \stackrel{\sim}{\longrightarrow} \mathcal{S}^{\bullet}\right|_{U_{j}}
$$

For simplicity we denote $E_{U_{i}}^{\bullet}$ by $E_{i}^{\bullet}$ and $U_{i} \cap U_{j}$ by $U_{i j}$. Hence on $U_{i j}$ we have

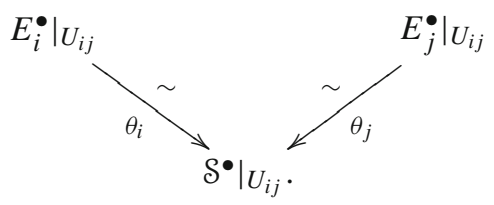

Since $E_{i}^{\bullet}$ and $E_{j}^{\bullet}$ are bounded and locally free, we can refine the open cover if necessary and lift the identity map on $\left.\mathcal{S}^{\bullet}\right|_{U_{i j}}$ (under some assumptions on $\mathcal{S}^{\bullet}$, see Lemma 2.30 below) to a map $a_{j i}: E_{j}^{\bullet} \rightarrow E_{i}^{\bullet}$, i.e. the following diagram:

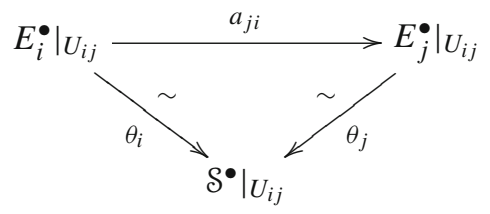

commutes up to homotopy. 
It is expected that the maps $a_{j i}$ play the role of transition functions, but we will show that they do not. Consider a third open subset $U_{k}$ together with $E_{k}^{\bullet}$ on it. According to the discussion above, the following diagram:

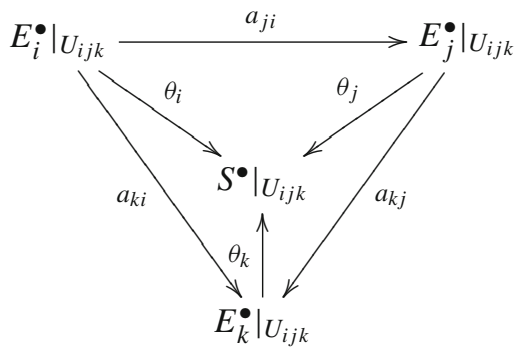

commutes up to homotopy. More precisely, we have a degree -1 map $a_{k j i}: E_{i}^{\bullet} \rightarrow$ $E_{k}^{\bullet-1}$ on $U_{i j k}$ such that

$$
a_{k i}-a_{k j} a_{j i}=\left[d, a_{k j i}\right],
$$

where $d$ is the differential on $E_{i}^{\bullet}$ and $E_{k}^{\bullet}$. In other words, the maps $a_{j i}$ only satisfy the cocycle condition up to homotopy. Hence we cannot simply use them to glue the sheaves $E_{i}^{\bullet}$ into a complex of sheaves on $X$. On the other hand, we expect that the homotopy operators $a_{k j i}$ satisfy compatible relations up to higher homotopies.

Toledo and Tong in [19] show that all these compatibility data together satisfy the Maurer-Cartan equation

$$
\delta a+a \cdot a=0
$$

which will be explained in Sect. 2. They call a collection $E_{i}^{\bullet}$ together with such maps $a$ a twisted complex or twisted cochain. In this paper we call it twisted perfect complex and keep the term twisted complex for a more general concept (for precise definition, see Sect. 2). Moreover, O'Brian, Toledo and Tong have proved that every perfect complex has a twisted resolution, see [15, Propoistion 1.2.3] or Proposition 3.20. This result is closely related to the essential-surjectivity of a dg-enhancement. Nevertheless, they have not attempted to build any equivalence of categories.

In this paper we construct a sheafification functor which is a dg-functor

$$
\mathcal{S}: \operatorname{Tw}_{\text {perf }}(X) \rightarrow \operatorname{Qcoh}_{\text {perf }}(X),
$$

where $\operatorname{Tw}_{\text {perf }}(X)$ denotes the dg-category of twisted perfect complexes on $X$ and $\mathrm{Qcoh}_{\text {perf }}(X)$ denotes the dg-category of perfect complexes of quasi-coherent sheaves on $X$.

We will prove that the dg-functor $\mathcal{S}$ gives the expected dg-enhancement, Theorem 3.29: Under reasonable conditions, the sheafification functor induces an equivalence of categories

$$
\mathcal{S}: \operatorname{HoTw}_{\text {perf }}(X) \rightarrow \mathrm{D}_{\text {perf }}(\mathrm{Q} \operatorname{coh}(X))
$$


We would also like to consider perfect complexes of general $\mathcal{O}_{X}$-modules rather than quasi-coherent modules. Actually we have, Theorem 3.32: Under some additional conditions, the sheafification functor induces an equivalence of categories

$$
\mathcal{S}: \operatorname{HoTw}_{\text {perf }}(X) \rightarrow \mathrm{D}_{\text {perf }}(X)
$$

Here we briefly mention the strategy of the proof. We extend the dg-category of twisted perfect complexes to a more general dg-category of twisted complexes on $X$ and define a twisting functor

$$
\mathcal{T}: \operatorname{Sh}(X) \rightarrow \operatorname{Tw}(X)
$$

which is also a dg-functor, where $\operatorname{Sh}(X)$ denotes the dg-category of sheaves on $X$ and $\operatorname{Tw}(X)$ denotes the dg-category of twisted complexes on $X$. The essential-surjectivity and fully-faithfulness of $\mathcal{S}$ can be achieved by a careful study of the relations between $\mathcal{S}$ and $\mathcal{T}$.

The constructions and proofs are inspired by [3, Section 4]. In fact Block gives a Dolbeault-theoretic dg-enhancement of perfect complexes in [3] while our construction can be considered as a Čech-theoretic enhancement.

Remark 1.2 In [12], Lunts and Schnürer introduced another dg-enhancement of the derived category of perfect complexes on a scheme and they call it the Čech enhancement. Conceptually the enhancement in [12] is very similar to the twisted complexes in this paper and we will discuss the relations between them in Sect. 3.4, Remark 3.34.

This paper is organized as follows. In Sect. 2 we give the definition of twisted (perfect) complexes. We show that these dg-categories have a pre-triangulated structure. Moreover we introduce weak equivalences between twisted complexes.

In Sect. 3 we construct the dg-enhancement. In more details, we construct the sheafification functor $\mathcal{S}$ in Sect. 3.1. In Sect. 3.2 we prove that the image of a twisted perfect complex under $\mathcal{S}$ is really a perfect complex. In Sect. 3.3 we prove that $\mathcal{S}$ is essentially surjective and in Sect. 3.4 we prove that $\mathcal{S}$ is fully faithful. Hence $\mathcal{S}$ gives the dg-enhancement.

In Sect. 4 we talk about some applications of twisted complexes. In particular we illustrate the application in descent theory.

In Sect. 5 we talk about some further topics. In Sect. 5.1 we introduce the twisted coherent complexes and prove that they form a dg-enhancement of the derived category of bounded above complexes of coherent sheaves. Actually the proofs are the same as those for twisted perfect complexes.

In Sect. 5.2 we make a digression and discuss the degenerate twisted complexes and show how they give splitting of idempotents.

In Sect. 5.3 we outline an alternative approach to this object: We wish to put a suitable model structure on twisted complexes and view $\mathcal{S}$ and $\mathcal{T}$ in terms of Quillen adjunctions.

In Appendix 1 we compare coherent complexes and pseudo-coherent complexes. Moreover we study the relation between quasi-coherent modules and general $\mathcal{O}_{X^{-}}$ modules. 
To ensure Theorem 3.29 we need that the open cover $\left\{U_{i}\right\}$ of $X$ is fine enough, in Appendix 2 we discuss good covers of a ringed space $X$.

\section{A review of twisted complexes}

\subsection{A quick review of perfect complexes}

Before talking about twisted complexes, we give a quick review of the derived category of perfect complexes and fix the notations in this subsection. For more details see [17, $20]$.

Definition 2.1 Let $\left(X, \mathcal{O}_{X}\right)$ be a locally ringed space. A complex $\mathcal{S}^{\bullet}$ is strictly perfect if $\mathcal{S}^{i}$ is zero for all but finitely many $i$ and $\mathcal{S}^{i}$ is a direct summand of a finite free $\mathcal{O}_{X}$-module for all $i$. The second condition is equivalent to that $\mathcal{S}^{i}$ is a finite locally free $\mathcal{O}_{X}$-module for all $i$.

Moreover, a complex $\mathcal{S}^{\bullet}$ of $\mathcal{O}_{X}$-modules is perfect if for any point $x \in X$, there exist an open neighborhood $U$ of $x$ and a strictly perfect complex $\mathcal{E}_{U}^{\bullet}$ on $U$ such that the restriction $\left.\mathcal{S}^{\bullet}\right|_{U}$ is isomorphic to $\mathcal{E}_{U}^{\bullet}$ in $\mathrm{D}\left(\mathcal{O}_{U}\right.$-mod), the derived category of sheaves of $\mathcal{O}_{X}$-modules on $U$.

Caution 2.2 If we did not assume that $X$ is a locally ringed space, then it may not be true that a direct summand of a finite free $\mathcal{O}_{X}$-module is finite locally free. See [20, Tag 08C3].

Remark 2.3 In fact, the definition of perfect complex is equivalent to the stronger requirement that for any point $x \in X$, there exist an open neighborhood $U$ of $x$ and a bounded complex of finite rank locally free sheaves $\mathcal{E}_{U}^{\bullet}$ on $U$ together with a quasi-isomorphism $\left.\mathcal{E}_{U}^{\bullet} \stackrel{\sim}{\longrightarrow} \mathcal{S}^{\bullet}\right|_{U}$. See [20, Tag 08C3, Lemma 20.38.8] for details.

Remark 2.4 It is obvious that a strictly perfect complex must be perfect. However, on a general ringed space $\left(X, \mathcal{O}_{X}\right)$ perfect complexes are not necessarily strictly perfect. In [12, Section 2.3], Lunts and Schnürer say that the scheme $X$ satisfies condition GSP if every perfect complex on $X$ is quasi-isomorphic to a strictly perfect complex. It can be proved that any affine scheme or projective scheme or separated regular Noetherian scheme satisfies condition GSP, see [2, Exposé II, Propositions 2.2.7 and 2.2.9] or [17, Example 2.1.2 and Proposition 2.3.1].

We consider the following categories.

Definition 2.5 Let $\mathrm{Sh}(X)$ be the dg-category of complexes of $\mathcal{O}_{X}$-modules on $X$. Let $\operatorname{Sh}_{\text {perf }}(X)$ be the full dg-subcategory of perfect complexes on $X$.

Let $\mathrm{K}(X)$ be the homotopy category of complexes of $\mathcal{O}_{X}$-modules on $X$. Then $\mathrm{K}_{\text {perf }}(X)$ is the triangulated subcategory of $\mathrm{K}(X)$ which consists of perfect complexes of $\mathcal{O}_{X}$-module.

Moreover let $\mathrm{D}(X)$ be the derived category of complexes of $\mathcal{O}_{X}$-modules on $X$. Then $\mathrm{D}_{\text {perf }}(X)$ is the triangulated subcategory of $\mathrm{D}(X)$ which consists of perfect complexes of $\mathcal{O}_{X}$-modules. 
We need also to consider the complexes of quasi-coherent sheaves on $X$ and we have the following definition.

Definition 2.6 Let $\mathrm{Qcoh}(X)$ be the dg-category of complexes of quasi-coherent sheaves on $X$. It is clear that $\mathrm{Q} \operatorname{coh}(X)$ is a full dg-subcategory of $\operatorname{Sh}(X)$. Let $\mathrm{Qcoh}_{\text {perf }}(X)$ be the full dg-subcategory of Qcoh $(X)$ which consists of perfect complexes of quasi-coherent sheaves. Qcoh perf $(X)$ is also a full dg-subcategory of $\operatorname{Sh}_{\text {perf }}(X)$.

Let $\mathrm{K}(\mathrm{Q} \operatorname{coh}(X))$ be the homotopy category of $\mathrm{Q} \operatorname{coh}(X)$ and $\mathrm{D}(\mathrm{Q} \operatorname{coh}(X))$ be its derived category. Similarly we have $\mathrm{K}_{\text {perf }}(\mathrm{Q} \operatorname{coh}(X))$ and $\mathrm{D}_{\text {perf }}(\mathrm{Q} \operatorname{coh}(X))$.

Remark 2.7 We have the natural inclusion $i: \mathrm{Q} \operatorname{coh}(X) \rightarrow \operatorname{Sh}(X)$ which induces a functor

$$
\widetilde{i}: \mathrm{D}(\mathrm{Q} \operatorname{coh}(X)) \rightarrow \mathrm{D}_{\mathrm{Q} \operatorname{coh}}(X),
$$

where $\mathrm{D}_{\mathrm{Qcoh}}(X)$ is the derived category of complexes of $\mathcal{O}_{X}$-modules with quasicoherent cohomologies. However, for general $\left(X, \mathcal{O}_{X}\right)$ the functor $\widetilde{i}$ is not necessarily essentially surjective nor fully faithful. As a result we need to distinguish complexes of quasi-coherent modules and complexes of general $\mathcal{O}_{X}$-modules. This issue will be discussed further in Appendix 1.

\subsection{Notations of bicomplexes and sign conventions}

In this subsection we introduce some notations which are necessary in the definition of twisted complexes, for reference see [14, Section 1].

Let $\left(X, \mathcal{O}_{X}\right)$ be a locally ringed space with paracompact underlying topological space and $\mathcal{U}=\left\{U_{i}\right\}$ be a locally finite open cover of $X$. Let $U_{i_{0} \ldots i_{n}}$ denote the intersection $U_{i_{0}} \cap \cdots \cap U_{i_{n}}$.

Remark 2.8 The papers $[14,15,19]$ focus on the special case that $X$ is a complex manifold and $\mathcal{O}_{X}$ is the sheaf of holomorphic functions on $X$. In this paper we consider more general $\left(X, \mathcal{O}_{X}\right)$.

For each $U_{i_{k}}$, let $E_{i_{k}}^{\bullet}$ be a graded sheaf of $\mathcal{O}_{X}$-modules on $U_{i_{k}}$. Let

$$
C^{\bullet}\left(\mathcal{U}, E^{\bullet}\right)=\prod_{p, q} C^{p}\left(\mathcal{U}, E^{q}\right)
$$

be the bigraded complexes of $E^{\bullet}$. More precisely, an element $c^{p, q}$ of $C^{p}\left(\mathcal{U}, E^{q}\right)$ consists of a section $c_{i_{0} \ldots i_{p}}^{p, q}$ of $E_{i_{0}}^{q}$ over each non-empty intersection $U_{i_{0} \ldots i_{p}}$. If $U_{i_{0} \ldots i_{p}}=$ $\varnothing$, simply let the component on it be zero.

Now if another graded sheaf $F_{i_{k}}^{\bullet}$ of $\mathcal{O}_{X}$-modules is given on each $U_{i_{k}}$, then we can consider the bigraded complex

$$
C^{\bullet}\left(\mathcal{U}, \operatorname{Hom}^{\bullet}(E, F)\right)=\prod_{p, q} C^{p}\left(\mathcal{U}, \operatorname{Hom}^{q}(E, F)\right) .
$$


An element $u^{p, q}$ of $C^{p}\left(\mathcal{U}, \operatorname{Hom}^{q}(E, F)\right)$ gives a section $u_{i_{0} \ldots i_{p}}^{p, q}$ of $\operatorname{Hom}_{\mathcal{O}_{X}-\operatorname{Mod}}^{q}\left(E_{i_{p}}^{\bullet}\right.$, $\left.F_{i_{0}}^{\bullet}\right)$, i.e. a degree $q$ map from $E_{i_{p}}^{\bullet}$ to $F_{i_{0}}^{\bullet}$ over the non-empty intersection $U_{i_{0} \ldots i_{p}}$. Notice that we require $u^{p, q}$ to be a map from $F^{\bullet}$ on the last subscript of $U_{i_{0} \ldots i_{p}}$ to $E^{\bullet}$ on the first subscript of $U_{i_{0} \ldots i_{p}}$. Again, if $U_{i_{0} \ldots i_{p}}=\varnothing$, let the component on it be zero.

Remark 2.9 In this paper when we talk about degree $(p, q)$, the first index always indicates the Čech degree while the second index always indicates the graded sheaf degree.

We need to study the compositions of $C^{\bullet}\left(\mathcal{U}, \operatorname{Hom}^{\bullet}(E, F)\right)$. Let $\left\{G_{i_{k}}^{\bullet}\right\}$ be a third graded sheaf of $\mathcal{O}_{X}$-modules, then there is a composition map

$$
C^{\bullet}\left(\mathcal{U}, \operatorname{Hom}^{\bullet}(F, G)\right) \times C^{\bullet}\left(\mathcal{U}, \operatorname{Hom}^{\bullet}(E, F)\right) \rightarrow C^{\bullet}\left(\mathcal{U}, \operatorname{Hom}^{\bullet}(E, G)\right)
$$

In fact, for $u^{p, q} \in C^{p}\left(\mathcal{U}, \operatorname{Hom}^{q}(F, G)\right)$ and $v^{r, s} \in C^{r}\left(\mathcal{U}, \operatorname{Hom}^{s}(E, F)\right)$, their composition $(u \cdot v)^{p+r, q+s}$ is given by (see [14, Equation (1.1)])

$$
(u \cdot v)_{i_{0} \ldots i_{p+r}}^{p+r, q+s}=(-1)^{q r} u_{i_{0} \ldots i_{p}}^{p, q} v_{i_{p} \ldots i_{p+r}}^{r, s},
$$

where the right-hand side is the naïve composition of sheaf maps.

In particular, $C^{\bullet}\left(\mathcal{U}, \operatorname{Hom}^{\bullet}(E, E)\right)$ becomes an associative algebra under this composition (it is easy but tedious to check the associativity). We also notice that $C^{\bullet}\left(\mathcal{U}, E^{\bullet}\right.$ ) becomes a left module over this algebra. In fact the action

$$
C^{\bullet}\left(\mathcal{U}, \operatorname{Hom}^{\bullet}(E, E)\right) \times C^{\bullet}\left(\mathcal{U}, E^{\bullet}\right) \rightarrow C^{\bullet}\left(\mathcal{U}, E^{\bullet}\right)
$$

is given by $\left(u^{p, q}, c^{r, s}\right) \mapsto(u \cdot c)^{p+r, q+s}$ where the action is given by (see [14, Equation (1.2)])

$$
(u \cdot c)_{i_{0} \ldots i_{p+r}}^{p+r, q+s}=(-1)^{q r} u_{i_{0} \ldots i_{p}}^{p, q} c_{i_{p} \ldots i_{p+r}}^{r, s},
$$

where the right-hand side is given by evaluation.

There is also a Čech-style differential operator $\delta$ on $C^{\bullet}\left(\mathcal{U}, \operatorname{Hom}^{\bullet}(E, F)\right)$ and $C^{\bullet}\left(\mathcal{U}, E^{\bullet}\right)$ of bidegree $(1,0)$ given by the formula

$$
(\delta u)_{i_{0} \ldots i_{p+1}}^{p+1, q}=\left.\sum_{k=1}^{p}(-1)^{k} u_{i_{0} \ldots \hat{i}_{k} \ldots i_{p+1}}^{p, q}\right|_{U_{i_{0} \ldots i_{p+1}}}, \quad u^{p, q} \in C^{p}\left(\mathcal{U}, \operatorname{Hom}^{q}(E, F)\right),
$$

and

$$
(\delta c)_{i_{0} \ldots i_{p+1}}^{p+1, q}=\left.\sum_{k=1}^{p+1}(-1)^{k} c_{i_{0} \ldots \widehat{i}_{k} \ldots i_{p+1}}^{p, q}\right|_{U_{i_{0} \ldots i_{p+1}}}, \quad c^{p, q} \in C^{p}(\mathcal{U}, E) .
$$

Caution 2.10 Notice that the map $\delta$ defined above is different from the usual Čech differential. In (5) we do not include the 0 -th and the $(p+1)$-th indices and in (6) we do not include the 0 -th index.

By a routine check we have 
Proposition 2.11 The differential satisfies the Leibniz rule. More precisely,

$$
\begin{aligned}
& \delta(u \cdot v)=(\delta u) \cdot v+(-1)^{|u|} u \cdot(\delta v), \\
& \delta(u \cdot c)=(\delta u) \cdot c+(-1)^{|u|} u \cdot(\delta c),
\end{aligned}
$$

where $|u|$ is the total degree of $u$.

\subsection{The definition of twisted complex}

Now we can define twisted complexes.

Definition 2.12 Let $\left(X, \mathcal{O}_{X}\right)$ be a locally ringed, paracompact space and $\mathcal{U}=\left\{U_{i}\right\}$ be a locally finite open cover of $X$. A twisted complex consists of graded sheaves $E_{i}^{\bullet}$ of $\mathcal{O}_{X}$-modules on each $U_{i}$ together with a collection of morphisms

$$
a=\sum_{k \geqslant 0} a^{k, 1-k}
$$

where $a^{k, 1-k} \in C^{k}\left(\mathcal{U}, \operatorname{Hom}^{1-k}(E, E)\right)$ such that they satisfy the Maurer-Cartan equation $\delta a+a \cdot a=0$. More explicitly, for $k \geqslant 0$

$$
\delta a^{k-1,2-k}+\sum_{i=0}^{k} a^{i, 1-i} \cdot a^{k-i, 1-k+i}=0 .
$$

Moreover, we impose the following non-degenerate condition: for each $i$, the chain map

$$
a_{i i}^{1,0}:\left(E_{i}^{\bullet}, a_{i}^{0,1}\right) \rightarrow\left(E_{i}^{\bullet}, a_{i}^{0,1}\right)
$$

is chain homotopic to the identity map.

The twisted complexes on $\left(X, \mathcal{O}_{X},\left\{U_{i}\right\}\right)$ form a dg-category: the objects are the twisted complexes $\mathcal{E}=\left(E_{i}^{\bullet}, a\right)$ and the morphisms from $\mathcal{E}=\left(E_{i}^{\bullet}, a\right)$ to $\mathcal{F}=\left(F_{i}^{\bullet}, b\right)$ are $C^{\bullet}\left(\mathcal{U}, \operatorname{Hom}^{\bullet}(E, F)\right)$. The degree of a morphism is given by the total degree of $C^{\bullet}\left(\mathcal{U}, \operatorname{Hom}^{\bullet}(E, F)\right)$. Moreover, the differential of a morphism $\phi$ is given by

$$
d \phi=\delta \phi+b \cdot \phi-(-1)^{|\phi|} \phi \cdot a
$$

We denote the dg-category of twisted complexes on $\left(X, \mathcal{O}_{X},\left\{U_{i}\right\}\right)$ by $\operatorname{Tw}\left(X, \mathcal{O}_{X}\right.$, $\left.\left\{U_{i}\right\}\right)$. If there is no danger of confusion we can simply denote it by $\operatorname{Tw}(X)$. 
Actually the first few terms of the Maurer-Cartan equation (7) can be written as

$$
\begin{aligned}
a_{i}^{0,1} \cdot a_{i}^{0,1} & =0, \\
a_{i}^{0,1} \cdot a_{i j}^{1,0}+a_{i j}^{1,0} \cdot a_{j}^{0,1} & =0, \\
-a_{i k}^{1,0}+a_{i j}^{1,0} \cdot a_{j k}^{1,0}+a_{i}^{0,1} \cdot a_{i j k}^{2,-1}+a_{i j k}^{2,-1} \cdot a_{k}^{0,1} & =0,
\end{aligned}
$$

Let us explain the meaning of these equations. The first equation tells us that for each $i$, $\left(E_{i}^{\bullet}, a_{i}^{0,1}\right)$ is a chain complex. The second equation, together with the sign convention in (3), tells us that $a_{i j}^{1,0}$ gives a chain map $\left(E_{j}^{\bullet}, a_{j}^{0,1}\right) \rightarrow\left(E_{i}^{\bullet}, a_{i}^{0,1}\right)$. The third equation says that we have the cocycle condition

$$
a_{i k}^{1,0}=a_{i j}^{1,0} a_{j k}^{1,0}
$$

up to homotopy with the homotopy operator $a_{i j k}^{2,-1}$.

Caution 2.13 Notice that a twisted complex itself is not a complex of sheaves on $X$.

For our purpose we need the following smaller dg-categories.

Definition 2.14 A twisted perfect complex $\mathcal{E}=\left(E_{i}^{\bullet}, a\right)$ is the same as twisted complex except that each $E_{i}^{\bullet}$ is required to be a strictly perfect complex on $U_{i}$. The twisted perfect complexes form a dg-category and we denote it by $\operatorname{Tw}_{\text {perf }}\left(X, \mathcal{O}_{X},\left\{U_{i}\right\}\right)$ or simply $\operatorname{Tw}_{\text {perf }}(X)$. Obviously, $\operatorname{Tw}_{\text {perf }}(X)$ is a full dg-subcategory of $\operatorname{Tw}(X)$.

Remark 2.15 The twisted perfect complex in this paper is almost the same as the twisted cochain in [14]. The only difference between our definition and theirs is that we do not require that for any $i$

$$
a_{i i}^{1,0}=\mathrm{id}_{E_{i}^{\bullet}} \quad \text { on the nose. }
$$

Our definition guarantees that the mapping cone exists in the category $\operatorname{Tw}(X)$, see Definition 2.22.

Remark 2.16 We would like to mention some related topics here.

- Our construction is very similar to the twisted complex in [7]. For example both constructions involve the Maurer-Cartan equation. The main difference is that the differential of the Maurer-Cartan equation in Bondal and Kapranov's twisted complex is the differential in the dg-category, while our differential is the Čech differential $\delta$.

- The construction of twisted complexes is very similar to the dg-nerve as in [13, 1.3.1.6] or in [5, Definition 2.3]. It is worthwhile to find the deeper relations.

- We expect the dg-category $\operatorname{Tw}_{\text {perf }}(X)$ gives an explicit realization of the homotopy limit of $\mathrm{L}\left(U_{i}\right)$, the dg-categories of locally free finitely generated sheaves on $U_{i}$. This problem has been solved in the recent preprint [4] and the construction depends heavily on the simplicial resolution of dg-categories in [10]. 
Definition 2.17 For a fixed twisted complex $\left(E^{\bullet}, a\right)$, we can define an operator $\delta_{a}$ on $C^{\bullet}\left(\mathcal{U}, E^{\bullet}\right)$ of total degree 1 by

$$
\delta_{a} c=\delta c+a \cdot c .
$$

The Maurer-Cartan equation $\delta a+a \cdot a=0$ implies that $\delta_{a}^{2}=0$, i.e. $\delta_{a}$ is a differential on $C^{\bullet}\left(\mathcal{U}, E^{\bullet}\right)$. We have the same construction when we restrict to $\operatorname{Tw}_{\text {perf }}(X)$.

Remark 2.18 We see that the differential $\delta_{a}$ in Definition 2.17 is a twist of the differential $\delta$. This justifies the name "twisted complex".

\subsection{Further study of the non-degeneracy condition of twisted complexes}

Recall that for each $i$, the $(0,1)$ component $a_{i}^{0,1}: E_{i}^{n} \rightarrow E_{i}^{n+1}$ is a differential of $\mathcal{O}_{X}$-modules on $U_{i}$, hence we get a complex $\left(E_{i}^{n}, a_{i}^{0,1}\right)$ on $U_{i}$. Remember that the map is the dot multiplication of $a_{i}^{0,1}$ as in (4).

Now we consider the map $a_{i i}^{1,0}: E_{i}^{n} \rightarrow E_{i}^{n}$, the Maurer-Cartan equation (7) in the $k=1$ case tells us

$$
a_{i i}^{1,0} \cdot a_{i}^{0,1}+a_{i}^{0,1} \cdot a_{i i}^{1,0}=0
$$

Actually under the sign convention in (3), the above equation becomes

$$
a_{i i}^{1,0} a_{i}^{0,1}-a_{i}^{0,1} a_{i i}^{1,0}=0 .
$$

In other words, $a_{i i}^{1,0}$ gives a chain map $\left(E_{i}^{n}, a_{i}^{0,1}\right) \rightarrow\left(E_{i}^{n}, a_{i}^{0,1}\right)$.

Let us denote the homotopy category of complexes of $\mathcal{O}_{X}$-modules on $U_{i}$ by $\mathrm{K}\left(U_{i}\right)$. Then we have the following lemma.

Lemma 2.19 If the maps $a^{k, 1-k}$ satisfy the Maurer-Cartan equation, then the map $a_{i i}^{1,0}:\left(E_{i}^{n}, a_{i}^{0,1}\right) \rightarrow\left(E_{i}^{n}, a_{i}^{0,1}\right)$ is an idempotent map in the homotopy category $\mathrm{K}\left(U_{i}\right)$, i.e. $\left(a_{i i}^{1,0}\right)^{2}=a_{i i}^{1,0}$ up to chain homotopy.

Proof The $k=2$ case of the Maurer-Cartan equation (7) gives us

$$
-a_{i i}^{1,0}+a_{i i}^{1,0} \cdot a_{i i}^{1,0}+a_{i}^{0,1} \cdot a_{i i i}^{2,-1}+a_{i i i}^{2,-1} \cdot a_{i}^{0,1}=0 .
$$

Hence if we take $a_{i i i}^{2,-1}$ to be the homotopy operator, this immediately gives what we want.

For later purpose we need the following lemma.

Lemma 2.20 If the maps $a^{k, 1-k}$ satisfy the Maurer-Cartan equation, then the map $a_{i i}^{1,0}:\left(E_{i}^{n}, a_{i}^{0,1}\right) \rightarrow\left(E_{i}^{n}, a_{i}^{0,1}\right)$ is homotopic to the identity map if and only if it is homotopic invertible.

Proof By Lemma 2.19, we know that $\left(a_{i i}^{1,0}\right)^{2}=a_{i i}^{1,0}$ up to chain homotopy. Then the result is obvious.

We will discuss the non-degeneracy condition further in Sect. 5.2. 


\subsection{The pre-triangulated structure on $\operatorname{Tw}(X)$}

The dg-category $\operatorname{Tw}(X)$ has a natural shift-by-one functor and a mapping cone construction as follows.

Definition 2.21 Let $\mathcal{E}=\left(E_{i}^{\bullet}, a\right)$ be a twisted complex. We define its shift $\mathcal{E}[1]$ to be $\mathcal{E}[1]=\left(E[1]_{i}^{\bullet}, a[1]\right)$, where

$$
E[1]_{i}^{\bullet}=E_{i}^{\bullet+1}, \quad a[1]^{k, 1-k}=(-1)^{k-1} a^{k, 1-k} .
$$

Moreover, let $\phi: \mathcal{E} \rightarrow \mathcal{F}$ be a morphism. We define its shift $\phi[1]$ as

$$
\phi[1]^{p, q}=(-1)^{q} \phi^{p, q} .
$$

Definition 2.22 Let $\phi^{\bullet,-\bullet}$ be a closed degree zero map between twisted complexes $\mathcal{E}=\left(E^{\bullet}, a^{\bullet, 1-\bullet}\right)$ and $\mathcal{F}=\left(F^{\bullet}, b^{\bullet, 1-\bullet}\right)$, we can define the mapping cone $\mathcal{G}=(G, c)$ of $\phi$ as follows (see [15, Section 1.1]):

$$
G_{i}^{n}=E_{i}^{n+1} \oplus F_{i}^{n}
$$

and

$$
c_{i_{0} \ldots i_{k}}^{k, 1-k}=\left(\begin{array}{cc}
(-1)^{k-1} a_{i_{0} \ldots i_{k}}^{k, 1-k} & 0 \\
(-1)^{k} \phi_{i_{0} \ldots i_{k}}^{k,-k} & b_{i_{0} \ldots i_{k}}^{k, 1-k}
\end{array}\right) .
$$

Remark 2.23 As a special case of (8) we get

$$
c_{i i}^{1,0}=\left(\begin{array}{cc}
a_{i i}^{1,0} & 0 \\
-\phi_{i i}^{1,-1} & b_{i i}^{1,0}
\end{array}\right) .
$$

It is clear that $c_{i i}^{1,0} \neq \mathrm{id}$ even if both $a_{i i}^{1,0}$ and $b_{i i}^{1,0}$ are equal to id since we cannot assume that $\phi_{i i}^{1,-1}=0$ for any $i$. This is the main technical reason that we drop the requirement $a_{i i}^{1,0}=\mathrm{id}$ in the definition of twisted complex, see Remark 2.15.

Nevertheless, we can prove that the mapping cone satisfies the non-degeneracy condition in Definition 2.1.

Lemma 2.24 Let $\phi^{\bullet,-} \bullet$ be a closed degree zero map between twisted complexes $\mathcal{E}=$ $\left(E^{\bullet}, a^{\bullet, 1-\bullet}\right)$ and $\mathcal{F}=\left(F^{\bullet}, b^{\bullet, 1-\bullet}\right)$. Let $\mathcal{G}=(G, c)$ be the mapping cone of $\phi$. Then

$$
c_{i i}^{1,0}:\left(G_{i}^{\bullet}, c_{i}^{0,1}\right) \rightarrow\left(G_{i}^{\bullet}, c_{i}^{0,1}\right)
$$

is chain homotopic to id. 
Proof By Lemma 2.20, we know that $a_{i i}^{1,0}$ and $b_{i i}^{1,0}$ are homotopic invertible, hence

$$
c_{i i}^{1,0}=\left(\begin{array}{cc}
a_{i i}^{1,0} & 0 \\
-\phi_{i i}^{1,-1} & b_{i i}^{1,0}
\end{array}\right)
$$

is also homotopic invertible since it is a lower block triangular matrix.

On the other hand, the maps $c^{k, 1-k}$ satisfy the Maurer-Cartan equation. Again by Lemma 2.20 we know that $c_{i i}^{1,0}$ is chain homotopic to id.

One can easily check the following result.

Proposition 2.25 $\mathrm{Tw}(X)$ is a pre-triangulated dg-category and $\mathrm{Tw}_{\mathrm{perf}}(X)$ is a pre-triangulated dg-subcategory of $\operatorname{Tw}(X)$. Therefore the category $\operatorname{HoTw}(X)$ is triangulated and $\operatorname{HoTw}_{\text {perf }}(X)$ is a triangulated subcategory of $\operatorname{HoTw}(X)$.

Caution 2.26 The degree and sign convention in the definition of mapping cones in this paper are slightly different to those in [15, Section 1.1].

\subsection{Weak equivalences in $\operatorname{Tw}(X)$}

In this subsection we specify the class of weak equivalences in $\operatorname{Tw}(X)$, which is very important in our later constructions.

Definition 2.27 Let $\mathcal{E}=\left(E^{\bullet}, a^{\bullet}, 1-\bullet\right)$ and $\mathcal{F}=\left(F^{\bullet}, b^{\bullet}, 1-\bullet\right)$ be two objects in $\operatorname{Tw}(X)$. A morphism $\phi: \mathcal{E} \rightarrow \mathcal{F}$ is called a weak equivalence if it satisfies the following two conditions:

- $\phi$ is closed and of degree zero;

- its $(0,0)$ component

$$
\phi_{i}^{0,0}:\left(E_{i}^{\bullet}, a_{i}^{0,1}\right) \rightarrow\left(F_{i}^{\bullet}, b_{i}^{0,1}\right)
$$

is a quasi-isomorphism of complexes of $\mathcal{O}_{X}$-modules on $U_{i}$ for each $i$.

Remark 2.28 The definition of weak equivalence between twisted complexes is first introduced in [9].

If $\mathcal{E}$ and $\mathcal{F}$ are both in the subcategory $\operatorname{Tw}_{\text {perf }}(X)$ we have a further result on weak equivalence between them. For this we need some assumption on the open cover $\left\{U_{i}\right\}$ and some technical lemmas, which we introduce here.

Lemma 2.29 Let $U$ be a subset of $X$ which satisfies $H^{k}(U, \mathcal{F})=0$ for any quasicoherent sheaf $\mathcal{F}$ on $U$ and any $k \geqslant 1$. Let $E^{\bullet}$ be a bounded above complex of finitely generated locally free sheaves on $U$ and $G^{\bullet}$ be an acyclic complex of quasi-coherent modules on $U$, then the Hom complex $\operatorname{Hom}^{\bullet}(E, G)$ is acyclic. 
Proof We have a filtration on $\operatorname{Hom}^{\bullet}(E, G)$ given by the $E^{\bullet}$ degree. More explicitly, let

$$
F^{k} \operatorname{Hom}^{\bullet}(E, G)=\left\{\phi \in \operatorname{Hom}^{\bullet}(E, G): \phi(e)=0 \text { if } \operatorname{deg}(e)>-k\right\}
$$

By a simple spectral sequence argument, it is sufficient to prove that

$$
\left(F^{k} \operatorname{Hom}^{\bullet}(E, G) / F^{k+1} \operatorname{Hom}^{\bullet}(E, G), d_{\mathrm{Hom}}\right)
$$

is acyclic for each $k$. We notice that

$$
\left(F^{k} \operatorname{Hom}^{\bullet}(E, G) / F^{k+1} \operatorname{Hom}^{\bullet}(E, G), d_{\mathrm{Hom}}\right) \cong\left(\operatorname{Hom}\left(E^{k}, G^{\bullet}\right), d_{G}\right) .
$$

We know that $\left(G^{\bullet}, d_{G}\right)$ is acyclic. On the other hand, $E^{k}$ is locally free finitely generated hence the assumption in the lemma guarantees that $\operatorname{Hom}\left(E^{k},-\right)$ is an exact functor, hence we get the acyclicity of $\operatorname{Hom}^{\bullet}(E, G)$.

Lemma 2.30 Let $U$ be a subset of $X$ which satisfies $H^{k}(U, \mathcal{F})=0$ for any quasicoherent sheaf $\mathcal{F}$ on $U$ and any $k \geqslant 1$. Suppose we have chain maps $r: E^{\bullet} \rightarrow F^{\bullet}$ and $s: G^{\bullet} \rightarrow F^{\bullet}$ between complexes of sheaves on $U$, where $E^{\bullet}$ is a bounded above complex of finitely generated locally free sheaves, and $F^{\bullet}$ and $G^{\bullet}$ are quasi-coherent. Moreover $s$ is a quasi-isomorphism. Then $r$ factors through $s$ up to homotopy, i.e. there exists a chain map $r^{\prime}: E^{\bullet} \rightarrow G^{\bullet}$ such that $s$ or $r^{\prime}$ is homotopic to $r$.

Proof We can take the mapping cone of $s$, which is acyclic, then the result is a simple corollary of Lemma 2.29.

With these lemmas we have the following result for twisted perfect complexes.

Proposition 2.31 Let the cover $\left\{U_{i}\right\}$ satisfy $H^{k}\left(U_{i}, \mathcal{F}\right)=0$ for any $i$, any quasicoherent sheaf $\mathcal{F}$ on $U_{i}$ and any $k \geqslant 1$. If $\mathcal{E}$ and $\mathcal{F}$ are both in the subcategory $\operatorname{Tw}_{\text {perf }}(X)$, then a closed degree zero morphism $\phi$ between twisted complexes $\mathcal{E}$ and $\mathcal{F}$ is a weak equivalence if and only if $\phi$ is invertible in the homotopy category $\operatorname{HoTw}_{\text {perf }}(X)$.

Proof It is obvious that homotopy invertibility implies weak equivalence. For the other direction, we know $\phi$ is a weak equivalence, hence $\phi_{i}^{0,0}: E_{i}^{\bullet} \rightarrow F_{i}^{\bullet}$ is a quasiisomorphism for each $i$. Since $F_{i}^{\bullet}$ is a bounded complex of finitely generated locally free sheaves, we apply Lemma 2.30 and get

$$
\psi_{i}^{0,0}: F_{i}^{\bullet} \rightarrow E_{i}^{\bullet}
$$

such that $\phi_{i}^{0,0} \circ \psi_{i}^{0,0}$ is homotopic to $\operatorname{id}_{F_{i}^{\bullet}}$. It is clear that $\psi_{i}^{0,0}$ is also a quasi-isomorphism and gives the two-side homotopy inverse of $\phi_{i}^{0,0}$.

The remaining task is to extend $\psi_{i}^{0,0}$ to a degree zero cocycle $\psi^{\bullet,-\bullet}$ in $\operatorname{Tw}(X)$ and to show that it gives the homotopy inverse of $\phi^{\bullet},-\bullet$. This is a simple spectral sequence argument which is the same as the proof of [3, Proposition 2.9]. 
Remark 2.32 The result of Proposition 2.31 is no longer true if one of $\mathcal{E}$ and $\mathcal{F}$ is not a twisted perfect complex.

We also have the following result.

Proposition 2.33 Let $\left\{U_{i}\right\}$ be an open cover of $X$ such that for any finite intersection $U_{I}$ we have $H^{k}\left(U_{I}, \mathcal{F}\right)=0$ for any quasi-coherent sheaf $\mathcal{F}$ on $U_{I}$ and any $k \geqslant 1$. Let $\mathcal{E}$ be a twisted perfect complex and $\mathcal{F}, \mathcal{G}$ be twisted complexes consisting of quasicoherent sheaves on each $U_{i}$. Let $\varphi: \mathcal{G} \rightarrow \mathcal{F}$ be a weak equivalence. Then any closed morphism $\phi: \mathcal{E} \rightarrow \mathcal{F}$ factors through $\varphi$ up to homotopy, i.e. there exists a chain map $\theta: \mathcal{E} \rightarrow \mathcal{G}$ such that $\varphi \cdot \theta$ is homotopic to $\phi$.

Proof The proof is inspired by that of [15, Proposition 1.2.3], see also Proposition 3.20. First we fix the notation. Let $\mathcal{E}=\left(E_{i}^{\bullet}, a\right), \mathcal{F}=\left(F_{i}^{\bullet}, b\right)$, and $\mathcal{G}=\left(G_{i}^{\bullet}, c\right)$. Let $l$ be the degree of $\phi: \mathcal{E} \rightarrow \mathcal{F}$.

Since $\varphi: \mathcal{G} \rightarrow \mathcal{F}$ is a weak equivalence, we know that on each $U_{i}, \varphi^{0,0}: G_{i}^{\bullet} \rightarrow F_{i}^{\bullet}$ is a quasi-isomorphism of complexes of quasi-coherent sheaves. By Lemma 2.30, we know that $\phi_{i}^{0, l}: E_{i}^{\bullet} \rightarrow F_{i}^{\bullet+l}$ factors through $\varphi_{i}^{0,0}$ up to homotopy, i.e. there exist $\theta_{i}^{0, l}: E_{i}^{\bullet} \rightarrow G_{i}^{\bullet+l}$ and $\mu_{i}^{0, l-1}: E_{i}^{\bullet} \rightarrow F_{i}^{\bullet+l-1}$ such that

$$
\begin{aligned}
c_{i}^{0,1} \theta_{i}^{0, l}-\theta_{i}^{0, l} a_{i}^{0,1} & =0, \\
\varphi_{i}^{0,0} \theta_{i}^{0, l}-\phi_{i}^{0, l} & =b_{i}^{0,1} \mu_{i}^{0, l-1}-\mu_{i}^{0, l-1} a_{i}^{0,1} .
\end{aligned}
$$

Now we need to do the following two constructions:

- Extend $\theta_{i}^{0, l}$ to a closed map $\theta: \mathcal{E} \rightarrow \mathcal{G}$ between twisted complexes.

- Extend $\mu_{i}^{0, l-1}$ to a homotopy between $\varphi \cdot \theta$ and $\phi$.

In more details, on each $U_{i_{0} \ldots i_{k}}$ we need to find $\theta_{i_{0} \ldots i_{k}}^{k, l-k}: E_{i_{k}}^{\bullet} \rightarrow G_{i_{0}}^{\bullet+l-k}$ and $\mu_{i_{0} \ldots i_{k}}^{k, l-1-k}$ : $E_{i_{k}}^{\bullet} \rightarrow F_{i_{0}}^{\bullet+l-1-k}$ such that

$$
\begin{aligned}
\sum_{j=1}^{k-1}(-1)^{j} \theta_{i}^{k-1, l+1+1-k} & +\sum_{j=0}^{k} c_{i_{0} \ldots i_{j}}^{j, 1-j} \cdot \theta_{i_{j} \ldots i_{k}}^{k-j, l+j-k} \\
& -(-1)^{l} \sum_{j=0}^{k} \theta_{i_{0} \ldots i_{j}}^{j, l-j} \cdot a_{i_{j} \ldots i_{k}}^{k-j, 1+j-k}=0
\end{aligned}
$$

and

$$
\begin{aligned}
\sum_{j=0}^{k} \varphi_{i_{0} \ldots i_{j}}^{j,-j} \cdot \theta_{i_{j} \ldots i_{k}}^{k-j, l+j-k}-\phi_{i_{0} \ldots i_{k}}^{k, l-k} & \\
=\sum_{j=1}^{k-1}(-1)^{j} \mu_{i_{0} \ldots \hat{i_{j} \ldots i_{k}}}^{k-1, l-k} & +\sum_{j=0}^{k} b_{i_{0} \ldots i_{j}}^{j, 1-j} \cdot \mu_{i_{j} \ldots i_{k}}^{k-j, l-1+j-k} \\
& +(-1)^{l} \sum_{j=0}^{k} \mu_{i_{0} \ldots i_{j}}^{j, l-1-j} \cdot a_{i_{j} \ldots i_{k}}^{k-j, 1+j-k} .
\end{aligned}
$$


We use induction to find $\theta$ and $\mu$. First remember that $\theta^{0, l}$ and $\mu^{0, l-1}$ have already been achieved. Now assume that for any multi-index $I$ with cardinality $|I|<k+1$ we have found the $\theta$ and $\mu$ on $U_{I}$ and they satisfy (9) and (10) on $U_{I}$.

Then we need to find $\theta_{i_{0} \ldots i_{k}}^{k, l-k}$ and $\mu_{i_{0} \ldots i_{k}}^{k, l-1-k}$. To do this we consider the mapping cone of $\varphi$ and denote it by $\mathcal{S}=\left(S_{i}^{n}, s\right)$. By definition we know that $S_{i}^{n}=G_{i}^{n+1} \oplus F_{i}^{n}$ and $s$ are given by

$$
s_{i_{0} \ldots i_{k}}^{k, 1-k}=\left(\begin{array}{cc}
(-1)^{k-1} c_{i_{0} \ldots i_{k}}^{k, 1-k} & 0 \\
(-1)^{k} \varphi_{i_{0} \ldots i_{k}}^{k,-k} & b_{i_{0} \ldots i_{k}}^{k, 1-k}
\end{array}\right) .
$$

In particular, on each $U_{i}$ we have

$$
s_{i}^{0,1}=\left(\begin{array}{cc}
-c_{i}^{0,1} & 0 \\
\varphi_{i}^{0,0} & b_{i}^{0,1}
\end{array}\right) .
$$

Since $\varphi: \mathcal{G} \rightarrow \mathcal{F}$ is a weak equivalence, we know that for each $U_{i},\left(S_{i}^{\bullet}, s_{i}^{0,1}\right)$ is an acyclic complex of quasi-coherent sheaves. By $\operatorname{Lemma} 2.29, \operatorname{Hom}^{\bullet}\left(E_{i}^{\bullet}, S_{i}^{\bullet}\right)$ is also acyclic. Moreover, $\operatorname{Hom}^{\bullet}\left(E_{i_{k}}^{\bullet}, S_{i_{0}}^{\bullet}\right)$ is acyclic on $U_{i_{0} \ldots i_{k}}$.

Then we rearrange (9) and (10) and get the following equations:

$$
\begin{aligned}
\sum_{j=1}^{k-1}(-1)^{j} \theta_{i_{0} \ldots \hat{i_{j} \ldots i_{k}}}^{k-1, l+1-k} & +\sum_{j=1}^{k} c_{i_{0} \ldots i_{j}}^{j, 1-j} \cdot \theta_{i_{j} \ldots i_{k}}^{k-j, l+j-k} \\
& -(-1)^{l} \sum_{j=0}^{k-1} \theta_{i_{0} \ldots i_{j}}^{j, l-j} \cdot a_{i_{j} \ldots i_{k}}^{k-j, 1+j-k} \\
& =(-1)^{l} \theta_{i_{0} \ldots i_{k}}^{k, l-k} \cdot a_{i_{k}}^{0,1}-c_{i_{0}}^{0,1} \cdot \theta_{i_{0} \ldots i_{k}}^{k, l-k}
\end{aligned}
$$

and

$$
\begin{aligned}
& -\sum_{j=1}^{k} \varphi_{i_{0} \ldots i_{j}}^{j,-j} \cdot \theta_{i_{j} \ldots i_{k}}^{k-j, l+j-k}+\phi_{i_{0} \ldots i_{k}}^{k, l-k}+\sum_{j=1}^{k-1}(-1)^{j} \mu_{i_{0} \ldots i_{j} \ldots i_{k}}^{k-1, l-k} \\
& +\sum_{j=1}^{k} b_{i_{0} \ldots i_{j}}^{j, 1-j} \cdot \mu_{i_{j} \ldots i_{k}}^{k-j, l-1+j-k}+(-1)^{l} \sum_{j=0}^{k-1} \mu_{i_{0} \ldots i_{j}}^{j, l-1-j} \cdot a_{i_{j} \ldots i_{k}}^{k-j, 1+j-k} \\
& =\varphi_{i_{0}}^{0,0} \cdot \theta_{i_{0} \ldots i_{k}}^{k, l-k}-b_{i_{0}}^{0,1} \cdot \mu_{i_{0} \ldots i_{k}}^{k, l-1-k}-(-1)^{l} \mu_{i_{0} \ldots i_{k}}^{k, l-1-k} \cdot a_{i_{k}}^{0,1} .
\end{aligned}
$$

We denote the left-hand side of (11) and (12) by $\Theta$ and $\Xi$ respectively. Notice that $\Theta$ and $\Xi$ do not involve $\theta_{i_{0} \ldots i_{k}}^{k, l-k}$ and $\mu_{i_{0} \ldots i_{k}}^{k, l-1-k}$. Moreover, by induction assumption we can check that

$$
-c_{i_{0}}^{0,1} \cdot \Theta-(-1)^{l} \Theta \cdot a_{i_{k}}^{0,1}=0
$$


and

$$
\varphi_{i_{0}}^{0,0} \cdot \Theta+b_{i_{0}}^{0,1} \cdot \Xi-(-1)^{l} \Xi \cdot a_{i_{k}}^{0,1}=0
$$

In other words, $(\Theta, \Xi): E_{i_{k}}^{\bullet} \rightarrow S_{i_{0}}^{\bullet+l-k}$ is closed. Since $\operatorname{Hom}^{\bullet}\left(E_{i_{k}}^{\bullet}, S_{i_{0}}^{\bullet}\right)$ is acyclic, we can find

$$
\left(\theta_{i_{0} \ldots i_{k}}^{k, l-k}, \mu_{i_{0} \ldots i_{k}}^{k, l-1-k}\right): E_{i_{k}}^{\bullet} \rightarrow S_{i_{0}}^{\bullet+l-1-k}
$$

such that

$$
-c_{i_{0}}^{0,1} \cdot \theta_{i_{0} \ldots i_{k}}^{k, l-k}+(-1)^{l} \theta_{i_{0} \ldots i_{k}}^{k, l-k} \cdot a_{i_{k}}^{0,1}=\Theta
$$

and

$$
\varphi_{i_{0}}^{0,0} \cdot \theta_{i_{0} \ldots i_{k}}^{k, l-k}-b_{i_{0}}^{0,1} \cdot \mu_{i_{0} \ldots i_{k}}^{k, l-1-k}-(-1)^{l} \mu_{i_{0} \ldots i_{k}}^{k, l-1-k} \cdot a_{i_{k}}^{0,1}=\Xi
$$

In other words, (11) and (12) hold.

Remark 2.34 Propositions 2.31 and 2.33 are not explicitly given in $[14,15,19]$.

\section{Twisted complexes and the dg-enhancement of $D_{\text {perf }}(X)$}

\subsection{The sheafification functor $\mathcal{S}$}

In this section we come to our main topic in this paper. First we fix a locally finite open cover $\mathcal{U}=\left\{U_{i}\right\}$ of $X$. As we noticed in Caution 2.13, a twisted complex $\mathcal{E}=\left(E_{i}^{\bullet}, a\right)$ is not a complex of sheaves. Nevertheless in this subsection we associate a complex of sheaves to each twisted complex on $X$.

First we introduce a variation of notations in (1) and (2). Let $E_{i_{k}}^{\bullet}=\left\{E_{i_{k}}^{r}\right\}_{r \in \mathbb{Z}}$ be a graded sheaf of $\mathcal{O}_{X}$-modules on $U_{i_{k}}$ as before. For $V$ an open subset of $X$, let

$$
C^{\bullet}\left(\mathcal{U}, E^{\bullet} ; V\right)=\prod_{p, q} C^{p}\left(\mathcal{U}, E^{q} ; V\right)
$$

be the bigraded complex on $V$. More precisely, an element $c^{p, q}$ of $C^{p}\left(\mathcal{U}, E^{q} ; V\right)$ consists of a section $c_{i_{0} \ldots i_{p}}^{p, q}$ of $E_{i_{0}}^{q}$ over each non-empty intersection $U_{i_{0} \ldots i_{n}} \cap V$. If $U_{i_{0} \ldots i_{n}} \cap V=\varnothing$, let the component on $U_{i_{0} \ldots i_{n}} \cap V$ simply be zero.

Similarly if another graded sheaf $F_{i_{k}}^{\bullet}$ of $\mathcal{O}_{X}$-modules is given on each $U_{i_{k}}$, and $V$ is an open subset of $X$, we can consider the bigraded complex

$$
C^{\bullet}\left(\mathcal{U}, \operatorname{Hom}^{\bullet}(E, F) ; V\right)=\prod_{p, q} C^{p}\left(\mathcal{U}, \operatorname{Hom}^{q}(E, F) ; V\right) .
$$


An element $u^{p, q}$ of complex $C^{p}\left(\mathcal{U}, \operatorname{Hom}^{q}(E, F) ; V\right)$ gives a section $u_{i_{0} \ldots i_{p}}^{p, q}$ of $\operatorname{Hom}_{\mathcal{O}_{X} \text {-mod }}^{q}\left(E_{i_{p}}^{\bullet}, F_{i_{0}}^{\bullet}\right)$ over each non-empty intersection $U_{i_{0} \ldots i_{n}} \cap V$. If $U_{i_{0} \ldots i_{n}} \cap V=\varnothing$, let the component on $U_{i_{0} \ldots i_{n}} \cap V$ simply be zero.

Moreover, let $\mathcal{E}=\left(E_{i}^{\bullet}, a\right)$ be a twisted complex, recall that in Definition 2.17 we defined a differential

$$
\delta_{a}=\delta+a
$$

on $C^{\bullet}\left(\mathcal{U}, E^{\bullet}\right)$. Now let $V$ be an open subset of $X$, we can restrict $\delta_{a}$ to $V$ to get a differential on $C^{\bullet}\left(U, E^{\bullet} ; V\right)$.

With all these notations, we can introduce the following definition.

Definition 3.1 For a twisted complex $\mathcal{E}=\left(E_{i}^{\bullet}, a\right)$, we define the associated complex of sheaves $\mathcal{S}(\mathcal{E})$ as follows: for each $n$, the degree $n$ part $\mathcal{S}^{n}(\mathcal{E})$ is a sheaf on $X$ such that for any open subset $V$ of $X$

$$
\mathcal{S}^{n}(\mathcal{E})(V)=\prod_{p+q=n} C^{p}\left(\mathcal{U}, E^{q} ; V\right)
$$

The differential on $\mathcal{S}^{\bullet}(\mathcal{E})$ is defined to be the sheafification of $\delta_{a}=\delta+a$. More precisely, for each open subset $V$ of $X$, the differential

$$
\mathcal{S}^{n}(\mathcal{E})(V) \rightarrow \mathcal{S}^{n+1}(\mathcal{E})(V)
$$

is given by $\delta+a$ restricted to $V$. We still denote it by $\delta_{a}$ since there is no danger of confusion.

It is obvious that $\mathcal{S}^{n}(\mathcal{E})$ is a sheaf of $\mathcal{O}_{X}$-module for each $n$ and $\delta_{a}: \mathcal{S}^{n}(\mathcal{E}) \rightarrow$ $\mathcal{S}^{n+1}(\mathcal{E})$ is a map of $\mathcal{O}_{X}$-modules.

Now we turn to the morphisms. Let $\phi: \mathcal{E} \rightarrow \mathcal{F}$ be a degree $n$ morphism in $\operatorname{Tw}(X)$. We can define the associated sheaf morphism

$$
\mathcal{S}(\phi): \mathcal{S}^{\bullet}(\mathcal{E}) \rightarrow \mathcal{S}^{\bullet+n}(\mathcal{F})
$$

in the same spirit as Definition 3.1, i.e. by restricting to each of $C^{p}\left(\mathcal{U}, E^{q} ; V\right)$.

In fact we can view $\mathcal{S}^{\bullet}(\mathcal{E})$ in another way. For this we recall some definitions in sheaf theory. Let $\mathcal{F}$ be any sheaf of $\mathcal{O}_{X}$-modules on $X$ and $U$ be an open subset of $X$ with $j: U \rightarrow X$ being the inclusion map. We denote the restriction sheaf of $\mathcal{F}$ on $U$ by $\left.\mathcal{F}\right|_{U}$. The pushforward of $\left.\mathcal{F}\right|_{U}$ is denoted by $j_{*}\left(\left.\mathcal{F}\right|_{U}\right)$ and it will be a sheaf of $\mathcal{O}_{X}$-modules on $X$ again and we also denote it by $\left.\mathcal{F}\right|_{U}$ if there is no confusion.

Remark 3.2 We do not use the fancy pushforward $j$ ! in this paper.

Then we have

$$
\mathcal{S}^{n}(\mathcal{E})=\left.\prod_{p+q=n} E_{i_{0}}^{q}\right|_{U_{i_{0} \ldots i p}}
$$

as a sheaf and the differential $\delta_{a}=\delta+a$ and the morphism $\mathcal{S}(\phi)$ are defined likewise by restriction. In conclusion we have the following definition. 
Definition 3.3 The above construction defines a dg-fuctor $\mathcal{S}: \operatorname{Tw}(X) \rightarrow \operatorname{Sh}(X)$ and we call it the sheafification functor.

Remark 3.4 If the complexes $E_{i}^{\bullet}$ are bounded and the cover $\left\{U_{i}\right\}$ is locally finite, it is easy to see that the product in $\mathcal{S}^{n}(\mathcal{E})=\left.\prod_{p+q=n} E_{i_{0}}^{q}\right|_{U_{i_{0} \ldots i p}}$ is locally finite, hence the image of a twisted perfect complex under $\mathcal{S}$ actually consists of quasi-coherent sheaves. In other words, the sheafification functor restricts to $\operatorname{Tw}_{\text {perf }}(X)$ and gives

$$
\mathcal{S}: \operatorname{Tw}_{\text {perf }}(X) \rightarrow \mathrm{Q} \operatorname{coh}(X)
$$

Further study of the sheafification of twisted perfect complexes will be given in the next subsection.

\subsection{The sheafification of twisted perfect complexes}

Let $\mathcal{E}$ be a twisted perfect complex, we want to show that the associated complex of sheaves $\left(\mathcal{S}^{\bullet}(\mathcal{E}), \delta_{a}\right)$ is perfect. In fact in this subsection we will get a more general result. The next proposition, which is important in our work, says that locally $\left(\mathcal{S}^{\bullet}(\mathcal{E}), \delta_{a}\right)$ contains the same information as $\left(E_{j}^{\bullet}, a_{j}^{0,1}\right)$ for each $j$.

Proposition 3.5 (local property of $\mathcal{S}$ ) Let $\mathcal{E}=\left(E_{i}^{\bullet}, a\right)$ be a twisted complex and $\left(\mathcal{S}^{\bullet}(\mathcal{E}), \delta_{a}\right)$ be the associated complex of sheaves. Then for each $U_{j}$ the complex of sheaves $\left.\left(\mathcal{S}^{\bullet}(\mathcal{E}), \delta_{a}\right)\right|_{U_{j}}$ is chain homotopy equivalent to $\left(E_{j}^{\bullet}, a_{j}^{0,1}\right)$, i.e. we have two morphisms

$$
f:\left.\left(\mathcal{S}^{\bullet}(\mathcal{E}), \delta_{a}\right)\right|_{U_{j}} \rightarrow\left(E_{j}^{\bullet}, a_{j}^{0,1}\right), \quad g:\left.\left(E_{j}^{\bullet}, a_{j}^{0,1}\right) \rightarrow\left(\mathcal{S}^{\bullet}(\mathcal{E}), \delta_{a}\right)\right|_{U_{j}}
$$

such that

$$
f \circ g=\mathrm{id}_{E_{j}^{\bullet}} \text { and } g \circ f=\mathrm{id}_{\left.\mathcal{S} \bullet(\mathcal{E})\right|_{U_{j}}} \text { up to chain homotopy. }
$$

Proof The proof is long and involves several technical claims. First we can construct the chain map $f:\left(\mathcal{S}^{\bullet}(\mathcal{E})(V), \delta_{a}\right) \rightarrow\left(E_{j}^{\bullet}(V), a_{j}^{0,1}\right)$ for $V \subset U_{j}$ by projecting to the $(0, n)$ component. In more details, we know that

$$
\mathcal{S}^{n}(\mathcal{E})(V)=\prod_{p+q=n} C^{p}\left(\mathcal{U}, E^{q} ; V\right)
$$

The $(0, n)$ component $C^{0}\left(\mathcal{U}, E^{n} ; V\right)$ has a further decomposition

$$
C^{0}\left(\mathcal{U}, E^{n} ; V\right)=\prod_{i_{0}} E_{i_{0}}^{n}\left(V \cap U_{i_{0}}\right) .
$$

We also notice that $j$ appears in one of indexes $i_{0}$. Then $f:\left(\mathcal{S}^{\bullet}(\mathcal{E})(V), \delta_{a}\right) \rightarrow$ $\left(E_{j}^{\bullet}(V), a_{j}^{0,1}\right)$ is given by first projecting to the $(0, n)$ component and then projecting to the $j$ component. It is easy to see that $f$ is a chain map. 
The construction of the map in the opposite direction $g:\left(E_{j}^{\bullet}(V), a_{j}^{0,1}\right) \rightarrow$ $\left(\mathcal{S}^{\bullet}(\mathcal{E})(V), \delta_{a}\right)$ is more complicated. We first introduce the following auxiliary morphism:

$$
\epsilon_{i_{0} \ldots i_{p}}^{p}: E_{i_{0}}^{\bullet}\left(U_{i_{0} \ldots i_{p} j} \cap V\right) \rightarrow E_{i_{0}}^{\bullet}\left(U_{i_{0} \ldots i_{p}} \cap V\right)
$$

as

$$
\epsilon_{i_{0} \ldots i_{p}}^{p}=(-1)^{p} \mathrm{id}
$$

Sometimes we simply denote it by $\epsilon^{p}$. Since $V \subseteq U_{j}$, we have $U_{i_{0} \ldots i_{p}} \cap V \subseteq U_{i_{0} \ldots i_{p} j}$ hence the above formula makes sense.

Notice that the identity map $E_{i_{0}}^{n-p}\left(U_{i_{0} \ldots i_{p} j} \cap V\right) \rightarrow E_{i_{0}}^{n-p}\left(U_{i_{0} \ldots i_{p}} \cap V\right)$ shifts the Čech degree by -1 and hence we introduce the factor $(-1)^{p}$ to compensate it.

We have the following property of the maps $\epsilon^{\bullet}$.

Claim 3.6 The maps $\epsilon^{\bullet}$ anti-commute with $a$ and $\delta$. More precisely, for a multi-index $i_{0}, \ldots, i_{p+q}$, we have

$$
a_{i_{0} \ldots i_{p}}^{p, 1-p} \epsilon_{i_{p} \ldots i_{p+q}}^{q}=-\epsilon_{i_{0} \ldots i_{p+q}}^{p+q} a_{i_{0} \ldots i_{p}}^{p, 1-p},
$$

where both sides are considered as maps

$$
E_{i_{p}}^{\bullet}\left(U_{i_{p} \ldots i_{p+q} j} \cap V\right) \rightarrow E_{i_{0}}^{\bullet+1-p}\left(U_{i_{0} \ldots i_{p+q}} \cap V\right) .
$$

As for $\delta$, we introduce a map $\widetilde{\delta}$ on $U_{i_{0} \ldots i_{p} j} \cap V$ as

$$
(\widetilde{\delta} c)_{i_{0} \ldots i_{p} j}=\sum_{k=1}^{p}(-1)^{k} c_{i_{0} \ldots \widehat{i_{k}} \ldots i_{p} j}
$$

Then we have

$$
\delta \epsilon^{p}=-\epsilon^{p+1} \widetilde{\delta}
$$

Proof First we prove that (15) holds. Let $c \in E_{i_{p}}^{\bullet}\left(U_{i_{p} \ldots i_{p+q} j} \cap V\right)$ be with Čech degree $q+1$. By definition

$$
\epsilon_{i_{p} \ldots i_{p+q}}^{q} c=(-1)^{q} c \in E_{i_{p}}^{\bullet}\left(U_{i_{p} \ldots i_{p+q}} \cap V\right)
$$

has Čech degree $q$. Then according to the sign convention in (4) we have

$$
\begin{aligned}
a_{i_{0} \ldots i_{p}}^{p, 1-p} \epsilon_{i_{p} \ldots i_{p+q}}^{q} c & =(-1)^{q} a_{i_{0} \ldots i_{p}}^{p, 1-p} \cdot c \\
& =(-1)^{q}(-1)^{(1-p) q} a_{i_{0} \ldots i_{p}}^{p, 1-p} c=(-1)^{p q} a_{i_{0} \ldots i_{p}}^{p, 1-p} c
\end{aligned}
$$


On the other hand, we have

$$
a_{i_{0} \ldots i_{p}}^{p, 1-p} \cdot c=(-1)^{(1-p)(1+q)} a_{i_{0} \ldots i_{p}}^{p, 1-p} c
$$

hence

$$
\epsilon_{i_{0} \ldots i_{p+q}}^{p+q} a_{i_{0} \ldots i_{p}}^{p, 1-p} \cdot c=(-1)^{p+q}(-1)^{(1-p)(1+q)} a_{i_{0} \ldots i_{p}}^{p, 1-p} c=(-1)^{1+p q} a_{i_{0} \ldots i_{p}}^{p, 1-p} c .
$$

Comparing the two sides, we get

$$
a_{i_{0} \ldots i_{p}}^{p, 1-p} \epsilon_{i_{p} \ldots i_{p+q}}^{q}=-\epsilon_{i_{0} \ldots i_{p+q}}^{p+q} a_{i_{0} \ldots i_{p}}^{p, 1-p} .
$$

Equation (16) follows similarly and we leave it as an exercise.

We move on to the definition of $g$. Recall that

$$
\mathcal{S}^{n}(\mathcal{E})(V)=\prod_{p+q=n} C^{p}\left(\mathcal{U}, E^{q} ; V\right)=\prod_{p \geqslant 0} \prod_{i_{0} \ldots i_{p}} E_{i_{0}}^{n-p}\left(U_{i_{0} \ldots i_{p}} \cap V\right)
$$

and it is sufficient to define the projection of $g$ to each component. With the help of the map $\epsilon^{p}$ we define that projection to be

$$
\epsilon^{p} \circ a_{i_{0} \ldots i_{p} j}^{p+1,-p}: E_{j}^{n}(V) \rightarrow E_{i_{0}}^{n-p}\left(U_{i_{0} \ldots i_{p}} \cap V\right), \quad p \geqslant 0 .
$$

Claim 3.7 The map $g:\left(E_{j}^{\bullet}(V), a_{j}^{0,1}\right) \rightarrow\left(\mathcal{S}^{\bullet}(\mathcal{E})(V), \delta_{a}\right)$ defined above is a chain map.

Proof It is a consequence of the Maurer-Cartan equation

$$
\delta a^{k-1,2-k}+\sum_{i=0}^{k} a^{i, 1-i} \cdot a^{k-i, 1-k+i}=0
$$

together with the anti-commute properties in Lemma 3.6.

Now we need to prove that $f$ and $g$ satisfy the relations in equation (14). First it is obvious that

$$
f \circ g=a_{j j}^{1,0}:\left(E_{j}^{\bullet}(V), a_{j}^{0,1}\right) \rightarrow\left(E_{j}^{\bullet}(V), a_{j}^{0,1}\right) .
$$

By definition, $a_{j j}^{1,0}=\mathrm{id}_{E_{j}^{\bullet}}$ up to homotopy hence we get $f \circ g=\operatorname{id}_{E_{j}^{\bullet}}$ up to homotopy.

The other half is more complicated. We need to build a map $h: \mathcal{S}^{\bullet}(\mathcal{E})(V) \rightarrow$ $\mathcal{S}^{\bullet-1}(\mathcal{E})(V)$ such that

$$
g \circ f-\mathrm{id}=\delta_{a} h+h \delta_{a} .
$$


In fact we define $h$ as

$$
(h c)_{i_{0} \ldots i_{k}}=(-1)^{k} c_{i_{0} \ldots i_{k} j}
$$

Clearly $h$ is a sheaf map with degree -1 . Moreover we have

$$
\begin{aligned}
\left(\delta_{a} h c\right)_{i_{0} \ldots i_{k}} & =(\delta(h c))_{i_{0} \ldots i_{k}}+(a \cdot(h c))_{i_{0} \ldots i_{k}} \\
& =\sum_{l=1}^{k}(-1)^{l}(h c)_{i_{0} \ldots \hat{i_{l}} \ldots i_{k}}+\sum_{l=0}^{k} a_{i_{0} \ldots i_{l}}^{l, 1-l} \cdot(h c)_{i_{l} \ldots i_{k}} \\
& =\sum_{l=1}^{k}(-1)^{l}(-1)^{k-1} c_{i_{0} \ldots \widehat{i_{l} \ldots i_{k} j}}+\sum_{l=0}^{k} a_{i_{0} \ldots i_{l}}^{l, 1-l} \cdot(h c)_{i_{l} \ldots i_{k}} .
\end{aligned}
$$

For the second term $a_{i_{0} \ldots i_{l}}^{l, 1-l} \cdot(h c)_{i_{l} \ldots i_{k}}$ we need to be more careful. We know that $(h c)_{i_{l} \ldots i_{k}}$ has Čech degree $k-l$ hence

$$
\begin{aligned}
a_{i_{0} \ldots i_{l}}^{l, 1-l} \cdot(h c)_{i_{l} \ldots i_{k}} & =(-1)^{(1-l)(k-l)} a_{i_{0} \ldots i_{l}}^{l, 1-l} \circ(h c)_{i_{l} \ldots i_{k}} \\
& =(-1)^{(1-l)(k-l)}(-1)^{k-l} a_{i_{0} \ldots i_{l}}^{l, 1-l} \circ c_{i_{l} \ldots i_{k} j} \\
& =(-1)^{l k-l} a_{i_{0} \ldots i_{l}}^{l, 1-l} \circ c_{i_{l} \ldots i_{k} j} .
\end{aligned}
$$

In conclusion we have

$$
\left(\delta_{a} h c\right)_{i_{0} \ldots i_{k}}=\sum_{l=1}^{k}(-1)^{k+l-1} c_{i_{0} \ldots \widehat{i_{l} \ldots i_{k} j}}+\sum_{l=0}^{k}(-1)^{l k-l} a_{i_{0} \ldots i_{l}}^{l, 1-l} \circ c_{i_{l} \ldots i_{k} j}
$$

On the other hand, we have

$$
\begin{aligned}
\left(h \delta_{a} c\right)_{i_{0} \ldots i_{k}}=(-1)^{k}\left(\delta_{a} c\right)_{i_{0} \ldots i_{k} j}=(-1)^{k}[(\delta c)+(a \cdot c)]_{i_{0} \ldots i_{k} j} & \\
=(-1)^{k}\left[\sum_{l=1}^{k}(-1)^{l} c_{i_{0} \ldots \hat{i_{l}} \ldots i_{k} j}\right. & +(-1)^{k+1} c_{i_{0} \ldots i_{k}} \\
& \left.+\sum_{l=0}^{k} a_{i_{0} \ldots i_{l}}^{l, 1-l} \cdot c_{i_{l} \ldots i_{k} j}+a_{i_{0} \ldots i_{k} j}^{k+1,-k} \cdot c_{j}\right] \\
=(-1)^{k}\left[\sum_{l=1}^{k}(-1)^{l} c_{i_{0} \ldots \hat{i_{l} \ldots i_{k} j}}\right. & +(-1)^{k+1} c_{i_{0} \ldots i_{k}} \\
& +\sum_{l=0}^{k}(-1)^{(l-1)(k-l+1)} a_{i_{0} \ldots i_{l}}^{l, 1-l} \circ c_{i_{l} \ldots i_{k} j} \\
& \left.+a_{i_{0} \ldots i_{k} j}^{k+1,-k} \circ c_{j}\right]
\end{aligned}
$$




$$
\begin{aligned}
=\sum_{l=1}^{k}(-1)^{k+l} c_{i_{0} \ldots \hat{i}_{l} \ldots i_{k} j}-c_{i_{0} \ldots i_{k}} & +\sum_{l=0}^{k}(-1)^{l k+l+1} a_{i_{0} \ldots i_{l}}^{l, 1-l} \circ c_{i_{l} \ldots i_{k} j-c_{i_{0} \ldots i_{k}}} \\
& +(-1)^{k} a_{i_{0} \ldots i_{k} j}^{k+1,-k} \circ c_{j} .
\end{aligned}
$$

In short, we have

$$
\begin{aligned}
\left(h \delta_{a} c\right)_{i_{0} \ldots i_{k}}=\sum_{l=1}^{k}(-1)^{k+l} c_{i_{0} \ldots \hat{i_{l}} \ldots i_{k} j}-c_{i_{0} \ldots i_{k}} & +\sum_{l=0}^{k}(-1)^{l k+l+1} a_{i_{0} \ldots i_{l}}^{l, 1-l} \circ c_{i_{l} \ldots i_{k} j} \\
& +(-1)^{k} a_{i_{0} \ldots i_{k} j}^{k+1,-k} \circ c_{j} .
\end{aligned}
$$

Comparing the above equality and (17), we get

$$
\left[\delta_{a} h c+h \delta_{a} c\right]_{i_{0} \ldots i_{k}}=-c_{i_{0} \ldots i_{k}}+(-1)^{k} a_{i_{0} \ldots i_{k} j}^{k+1,-k} \circ c_{j} .
$$

Recall that $f c=c_{j}$ and

$$
g(f c)_{i_{0} \ldots i_{k}}=\epsilon^{k} a_{i_{0} \ldots i_{k} j}^{k+1,-k} \cdot c_{j}=(-1)^{k} a_{i_{0} \ldots i_{k} j}^{k+1,-k} \circ c_{j}
$$

hence we get the desired result

$$
\left[\delta_{a} h c+h \delta_{a} c\right]_{i_{0} \ldots i_{k}}=-c_{i_{0} \ldots i_{k}}+g(f c)_{i_{0} \ldots i_{k}} .
$$

This finishes the proof of Proposition 3.5.

The perfectness now is a direct corollary of Proposition 3.5.

Corollary 3.8 If $\mathcal{E}=\left(E^{\bullet}, a\right)$ is a twisted perfect complex, then the sheafification $\mathcal{S}^{\bullet}(\mathcal{E})$ is a perfect complex on $\left(X, \mathcal{O}_{X}\right)$. In other words, the sheafification functor $\mathcal{S}$ restricts to $\operatorname{Tw}_{\text {perf }}(X)$ and gives the following dg-functor $\mathcal{S}: \operatorname{Tw}_{\text {perf }}(X) \rightarrow \operatorname{Sh}_{\text {perf }}(X)$.

Proof Proposition 3.5 tells us that $\left.\mathcal{S}^{\bullet}(\mathcal{E})\right|_{U_{j}}$ is isomorphic to $\left(E_{j}^{\bullet}, a_{j}^{0,1}\right)$ in $\mathrm{K}\left(U_{j}\right)$ hence by definition it is perfect on $U_{j}$. Moreover this is true for any member $U_{j}$ of the open cover, therefore $\mathcal{S}^{\bullet}(\mathcal{E})$ is a perfect complex of sheaves on $\left(X, \mathcal{O}_{X}\right)$.

Remark 3.9 Corollary 3.8 together with Remark 3.4 tells us that actually we have a dg-functor $\mathcal{S}: \operatorname{Tw}_{\text {perf }}(X) \rightarrow \operatorname{Qcoh}_{\text {perf }}(X)$.

Another consequence of Proposition 3.5 is the following criterion of weak equivalence. Recall that by Definition 2.27 a closed degree zero morphism $\phi^{\bullet,-\bullet}: \mathcal{E} \rightarrow \mathcal{F}$ is called a weak equivalence if its $(0,0)$ component $\phi_{i}^{0,0}:\left(E_{i}^{\bullet}, a^{0,1}\right) \rightarrow\left(F_{i}^{\bullet}, b^{0,1}\right)$ is a quasiisomorphism of complexes of $\mathcal{O}_{X}$-modules on $U_{i}$ for each $i$.

Corollary 3.10 (criterion of weak equivalence) A degree 0 cocycle $\phi^{\bullet,-\bullet}: \mathcal{E} \rightarrow \mathcal{F}$ in $\operatorname{Tw}(X)$ is a weak equivalence if and only if its sheafification $\mathcal{S}(\phi): \mathcal{S}(\mathcal{E}) \rightarrow \mathcal{S}(\mathcal{F})$ is a quasi-isomorphism. 
Proof First we fix $U_{j}$. It is obvious that the quasi-isomorphism

$$
f:\left.\mathcal{S}^{\bullet}(\mathcal{E})\right|_{U_{j}} \stackrel{\sim}{\longrightarrow} E_{j}^{\bullet}
$$

is functorial hence we have the following commutative diagram:

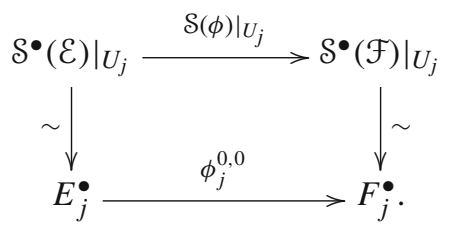

Now the claim is obviously true.

\subsection{The essential surjectivity of $\mathcal{S}$}

\subsubsection{The twisting functor $\mathcal{T}$ and some generalities}

Remark 3.9 ensures that we have the dg-functor

$$
S: \operatorname{Tw}_{\text {perf }}(X) \rightarrow \text { Qcoh }_{\text {perf }}(X)
$$

which induces an exact functor

$$
\mathcal{S}: \operatorname{HoTw}_{\text {perf }}(X) \rightarrow \mathrm{D}_{\text {perf }}(\mathrm{Q} \operatorname{coh}(X))
$$

In this subsection we will show that this functor is essentially surjective under some mild condition. Moreover we will show that the functor

$$
\mathcal{S}: \operatorname{HoTw}_{\text {perf }}(X) \rightarrow \mathrm{D}_{\text {perf }}(X)
$$

is essentially surjective under some additional conditions.

First we define a natural dg-functor from $\operatorname{Sh}(X)$ to $\operatorname{Tw}(X)$ as follows.

Definition 3.11 Let $\left(S^{\bullet}, d\right)$ be a complex of $\mathcal{O}_{X}$-modules. We define its associated twisted complex, $\mathcal{T}(S)$, by restricting to $U_{i}$. In more details, let $\left(E^{\bullet}, a\right)=\mathcal{T}(S)$ then

$$
E_{i}^{n}=\left.S^{n}\right|_{U_{i}}
$$

and

$$
a_{i}^{0,1}=\left.d\right|_{U_{i}}, \quad a_{i j}^{1,0}=\mathrm{id}, \quad a^{k, 1-k}=0 \text { for } k \geqslant 2 .
$$

The complex $\mathcal{T}$ of morphisms is defined in a similar way. We call the dg-functor $\mathcal{T}: \operatorname{Sh}(X) \rightarrow \operatorname{Tw}(X)$ the twisting functor. 
We would like to find the relation between the dg-functors $\mathcal{S}$ and $\mathcal{T}$. First we have the following result.

Proposition 3.12 Let $P=\left(S^{\bullet}, d\right)$ be a complex of $\mathcal{O}_{X}$-modules, the natural map

$$
\tau_{P}: P \rightarrow \mathcal{S T}(P)
$$

is a quasi-isomorphism. Hence $\tau:$ id $\rightarrow$ ST gives a natural isomorphism between functors (on the level of derived categories).

Proof By definition, $\mathcal{S T}(P)$ is the total complex of the double complex associated to $\mathcal{T}(P)$ and the map $\tau_{P}$ is given by the embedding into the 0 -th row of that double complex. Hence it is sufficient to prove that the Čech direction of the double complex is acyclic. But we know that the Čech complex (without taking global sections) is always acyclic.

On the other hand, let $\mathcal{E}=(E, a)$ be a twisted complex, we would like to define a closed degree 0 morphism

$$
\gamma_{\mathcal{E}}: \mathcal{T S}(\mathcal{E}) \rightarrow \mathcal{E}
$$

Actually for each $U_{i_{0} \ldots i_{p}}$ we need to construct a map

$$
\left(\gamma_{\mathcal{E}}\right)_{i_{0} \ldots i_{p}}^{p,-p}:\left.\mathcal{S}^{\bullet}(\mathcal{E})\right|_{U_{i_{p}}} \rightarrow E_{i_{0}}^{\bullet-p}
$$

Recall that $\mathcal{S}^{\bullet}(\mathcal{E})=\left.\prod_{j_{0} \ldots j_{k}} E_{j_{0}}^{\bullet-k}\right|_{U_{j_{0} \ldots j_{k}}}$, then $\left(\gamma_{\mathcal{E}}\right)_{i_{0} \ldots i_{p}}^{p,-p}$ is defined to be projecting to the component $i_{0} \ldots i_{p}$. In particular, $\left(\gamma_{\varepsilon}\right)_{j}^{0,0}$ is the map $f$ in Proposition 3.5. It is easy to verify that $\gamma_{\mathcal{E}}$ commutes with the differentials. From Proposition 3.5 we have

Proposition 3.13 The map $\gamma_{\mathcal{E}}: \mathcal{T S}(\mathcal{E}) \rightarrow \mathcal{E}$ is a weak equivalence.

Remark 3.14 If $\mathcal{E}$ is a twisted perfect complex, then $\mathcal{T} \mathcal{S}(\mathcal{E})$ is not necessarily a twisted perfect complex. Nevertheless, it is easy to see that $\mathcal{T} \mathcal{S}(\mathcal{E})$ consists of complexes of quasi-coherent sheaves on each $U_{i}$.

Proposition 3.15 Let $\mathcal{E}=(E, a)$ be a twisted complex, the composition

$$
\mathcal{S}(\mathcal{E}) \stackrel{\tau_{\mathcal{S}(\mathcal{E})}}{\longrightarrow} \mathcal{S} \mathcal{T} \mathcal{S}(\mathcal{E}) \stackrel{\mathcal{S}\left(\gamma_{\mathcal{E}}\right)}{\longrightarrow} \mathcal{S}(\mathcal{E})
$$

is equal to the identity map on $\mathcal{S}(\mathcal{E})$.

Proof The proof is just an untangling of definitions. By definition we know that

$$
\begin{aligned}
{[\mathcal{S T S}(\mathcal{E})]^{n} } & =\left.\prod_{p+q=n} \prod_{i_{0} \ldots i_{p}}\left[(\mathcal{T S}(\mathcal{E}))_{i_{0}}^{q}\right]\right|_{U_{i_{0} \ldots i_{p}}} \\
& =\left.\prod_{p+q=n} \prod_{i_{0} \ldots i_{p}} \prod_{s+t=q} \prod_{a_{0} \ldots a_{s}}\left(E_{a_{0}}^{t}||_{U_{a_{0} \ldots a_{S}}}\right)\right|_{U_{i_{0} \ldots i_{p}}}
\end{aligned}
$$


The map $\tau_{\mathcal{S}(\mathcal{E})}$ is the embedding into the 0-th row hence it maps the complex $\left.\prod_{s+t=n} \prod_{a_{0} \ldots a_{s}} E_{a_{0}}^{t}\right|_{U_{a_{0} \ldots a_{s}}}$ to the $p=0, q=n$ component of the above equation, i.e. $\left.\tau_{\mathcal{S}(\mathcal{E})} \operatorname{maps} \prod_{s+t=n} \prod_{a_{0} \ldots a_{s}} E_{a_{0}}^{t}\right|_{U_{a_{0} \ldots a_{S}}}$ to

$$
\left.\prod_{i_{0}} \prod_{s+t=n} \prod_{a_{0} \ldots a_{s}}\left(\left.E_{a_{0}}^{t}\right|_{U_{a_{0} \ldots a_{s}}}\right)\right|_{U_{i_{0}}}
$$

Then compose with $\mathcal{S}\left(\gamma_{\mathcal{E}}\right)$ and we get the identity map on $\left.\prod_{s+t=q} E_{a_{0}}^{t}\right|_{U_{a_{0} \ldots a_{s}}}$.

\subsubsection{The twisted resolution and the essential surjectivity on quasi-coherent sheaves}

Let $P=\left(S^{\bullet}, d\right)$ be a perfect complex. There is no guarantee that its associated twisted complex $\mathcal{T}(P)$ is a twisted perfect complex on the nose, even if we assume $P$ consists of quasi-coherent sheaves. Nevertheless we have a quasi-isomorphic result. First we need to introduce the following definitions.

Definition 3.16 A locally ringed space $\left(U, \mathcal{O}_{U}\right)$ is called $p$-good if it satisfies the following two conditions:

- For every perfect complex $\mathcal{P}^{\bullet}$ on $U$ which consists of quasi-coherent sheaves, there exist a strictly perfect complex $\mathcal{E}^{\bullet}$ on $U$ together with a quasi-isomorphism $u: \mathcal{E} \stackrel{\sim}{\longrightarrow} \mathcal{P} \bullet$.

- The higher cohomologies of quasi-coherent sheaves vanish, i.e. $H^{k}(U, \mathcal{F})=0$ for any quasi-coherent sheaf $\mathcal{F}$ on $U$ and any $k \geqslant 1$.

Remark 3.17 The letter "p" in the term "p-good space" stands for "perfect".

Then we can define p-good cover of a ringed space.

Definition 3.18 Let $\left(X, \mathcal{O}_{X}\right)$ be a locally ringed space, an open cover $\left\{U_{i}\right\}$ of $X$ is called a $p$-good cover if $\left(U_{I},\left.\mathcal{O}_{X}\right|_{U_{I}}\right)$ is a p-good space for any finite intersection $U_{I}$ of the open cover.

Remark 3.19 We introduce p-good covers mainly because we need to fix a cover which works for any complex of quasi-coherent sheaves on $X$. Actually a possible alternative way is to refine the open cover and consider the refinement of twisted complexes and get a direct limit

$$
\lim _{\longrightarrow} \text { refinement of }\left\{U_{i}\right\} \operatorname{Tw}\left(X, \mathcal{O}_{X},\left\{U_{i}\right\}\right)
$$

Nevertheless in this paper we do not take the above approach and just stick to a fixed p-good cover.

A lot of "reasonable" ringed spaces have p-good covers. For example we have

- $\left(X, \mathcal{O}_{X}\right)$ is a separated scheme, then any affine cover is p-good.

- $\left(X, \mathcal{O}_{X}\right)$ is a complex manifold with $\mathcal{O}_{X}$ the sheaf of holomorphic functions. In this case a Stein cover is p-good. 
- $\left(X, \mathcal{O}_{X}\right)$ is a paracompact topological space with soft structure sheaf $\mathcal{O}_{X}$. Then any contractible open cover is p-good.

Further discussions of p-good covers will be given in Appendix 2.

With the notion of p-good covers we can state and prove the following important proposition.

Proposition 3.20 (twisted resolution, see [15, Proposition 1.2.3]) Assume the cover $\left\{U_{i}\right\}$ is p-good. Let $P=\left(S^{\bullet}, d_{S}\right)$ be a perfect complex which consists of quasicoherent modules, then $\mathcal{T}(P)$ is weakly equivalent to a twisted perfect complex. More precisely there exist a twisted perfect complex $\mathcal{E}$ together with a weak equivalence (Definition 2.27)

$$
\phi: \mathcal{E} \stackrel{\sim}{\longrightarrow} \mathcal{T}(P)
$$

Proof This proposition and its proof are essentially the same as [15, Proposition 1.2.3]. For completeness we give the proof here in our terminology.

First we know that for each perfect complex $P=\left(S^{\bullet}, d_{S}\right)$, there exist a strictly perfect complex $E_{i}^{\bullet}$ on each $U_{i}$ together with a quasi-isomorphism

$$
\phi_{i}^{0,0}:\left.E_{i}^{\bullet} \stackrel{\sim}{\longrightarrow} S^{\bullet}\right|_{U_{i}}
$$

Let us denote the differential of the chain complex $E_{i}^{\bullet}$ by $a_{i}^{0,1}$. Now we need to do the following two constructions:

- Find maps $a^{k, 1-k}$ for $k \geqslant 1$ such that they and $a_{i}^{0,1}$ together make $E_{i}^{\bullet}$ a twisted complex.

- Extend the map $\phi_{i}^{0,0}$ to get a morphism $\left(E^{\bullet}, a\right) \rightarrow \mathcal{T}(P)$ in $\operatorname{Tw}(X)$.

Actually we can construct the two kinds of maps simultaneously. Let $L_{i}^{\bullet}$ be the mapping cone of $\phi_{i}^{0,0}$ (so far $L_{i}^{\bullet}$ is not the mapping cone of any twisted complexes), which is a complex of (not necessarily locally free) sheaves on each open cover $U_{i}$ and we denote its differential by $A_{i}^{0,1}$. In fact we have

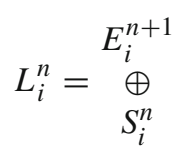

and

$$
A_{i}^{0,1}=\left(\begin{array}{cc}
-a_{i}^{0,1} & 0 \\
\phi_{i}^{0,0} & \left.d_{S}\right|_{U_{i}}
\end{array}\right) .
$$

We want to construct $A^{k, 1-k}$ in $C^{k}\left(\mathcal{U}, \operatorname{Hom}^{1-k}(L, L)\right)$ which make $L$ into a twisted complex. Moreover, we want $(L, A)$ to be the mapping cone of a closed degree zero morphism $\phi: \mathcal{E} \rightarrow \mathcal{T}(P)$ which extends $\phi_{i}^{0,0}$. More precisely, we have the following two requirements on $A^{k, 1-k}$ : 
- A satisfies the Maurer-Cartan equation

$$
\delta A+A \cdot A=0 .
$$

- We have

$$
A_{i}^{0,1}=\left(\begin{array}{cc}
-a_{i}^{0,1} & 0 \\
\phi_{i}^{0,0} & \left.d_{S}\right|_{U_{i}}
\end{array}\right), \quad A_{i j}^{1,0}=\left(\begin{array}{cc}
* & 0 \\
\left.* \mathrm{id}\right|_{U_{i j}}
\end{array}\right)
$$

and for $k \geqslant 2, A^{k, 1-k}$ is of the form $\left(\begin{array}{c}* 0 \\ * 0\end{array}\right)$.

The construction involves Lemmas 2.29 and 2.30 .

Notice that $S^{n}$ is quasi-coherent for each $n$, we apply Lemma 2.30 to the case $U=U_{i j}, r=\phi_{j}^{0,0}:\left.\left.E_{j}^{\bullet}\right|_{U_{i j}} \rightarrow S^{\bullet}\right|_{U_{i j}}$ and $s=\phi_{i}^{0,0}:\left.\left.E_{i}^{\bullet}\right|_{U_{i j}} \rightarrow S^{\bullet}\right|_{U_{i j}}$ and we obtain a chain map $r^{\prime}:\left.\left.E_{j}^{\bullet}\right|_{U_{i j}} \rightarrow E_{i}^{\bullet}\right|_{U_{i j}}$ together with a homotopy $h:\left.\left.E_{j}^{\bullet}\right|_{U_{i j}} \rightarrow S^{\bullet-1}\right|_{U_{i j}}$ such that

$$
\phi_{i}^{0,0} r^{\prime}-\phi_{j}^{0,0}=d_{S} h+h a_{j}^{0,1}
$$

Hence we get

$$
a_{i j}^{1,0}=r^{\prime}, \quad \phi_{i j}^{1,-1}=h .
$$

Moreover let

$$
A_{i j}^{1,0}=\left(\begin{array}{cc}
a_{i j}^{1,0} & 0 \\
-\phi_{i j}^{1,-1} & \left.\mathrm{id}\right|_{U_{i j}}
\end{array}\right)
$$

It is clear that $A^{1,0}$ satisfies

$$
A^{1,0} \cdot A^{0,1}+A^{0,1} \cdot A^{1,0}=0 .
$$

The maps $A^{k, 1-k}$ for $k \geqslant 2$ are constructed by induction: Let $D$ denote the differential on $\operatorname{Hom}^{\bullet}\left(L_{i_{k}}^{\bullet}, L_{i_{0}}^{\bullet}\right)$. We need to find $A_{i_{0} \ldots i_{k}}^{k, 1-k}$ on $U_{i_{0} \ldots i_{k}}$ satisfying

(i)

$$
(-1)^{k+1} D\left(A_{i_{0} \ldots i_{k}}^{k, 1-k}\right)=\left[\delta A^{k-1,2-k}+\sum_{l=1}^{k-1} A^{l, 1-l} \cdot A^{k-l, 1+l-k}\right]_{i_{0} \ldots i_{k}} .
$$

(ii) $A_{i_{0} \ldots i_{k}}^{k, 1-k}$ vanishes on the component $\left.S^{\bullet}\right|_{U_{i_{k}}}$ of $L_{i_{k}}^{\bullet}$.

Keep in mind that $L_{i}^{n}=E_{i}^{n+1} \oplus S_{i}^{n}$, condition (ii) is equivalent to the fact that $A_{i_{0} \ldots i_{k}}^{k, 1-k}$ lies in the subcomplex $\operatorname{Hom}^{\bullet}\left(E_{i_{k}}^{\bullet+1}, L_{i_{0}}^{\bullet}\right)$ of $\operatorname{Hom}^{\bullet}\left(L_{i_{k}}^{\bullet}, L_{i_{0}}^{\bullet}\right)$.

It is easy to verify that $\left[\delta A^{1,0}+A^{1,0} \cdot A^{1,0}\right]_{i j k}$ lies in $\operatorname{Hom}^{\bullet}\left(E_{i_{k}}^{\bullet+1}, L_{i_{0}}^{\bullet}\right)$. Hence by induction we know that the right-hand side of (18), $\left[\delta A^{k-1,2-k}+\sum_{l=1}^{k-1} A^{l, 1-l}\right.$. 
$\left.A^{k-l, 1+l-k}\right]_{i_{0} \ldots i_{k}}$, lies in $\operatorname{Hom}^{\bullet}\left(E_{i_{k}}^{\bullet+1}, L_{i_{0}}^{\bullet}\right)$ for $k \geqslant 2$. Also by induction we can show that it is a cocycle under the differential $D$. By $\operatorname{Lemma} 2.29, \operatorname{Hom}^{\bullet}\left(E_{i_{k}}^{\bullet+1}, L_{i_{0}}^{\bullet}\right)$ is acyclic, hence $A_{i_{0} \ldots i_{k}}^{k, 1-k}$ in $\operatorname{Hom}^{\bullet}\left(E_{i_{k}}^{\bullet+1}, L_{i_{0}}^{\bullet}\right)$ which satisfies (18) exists. By induction we have constructed the desired $(L, A)$.

With the help of Proposition 3.20 we can prove the essential surjectivity of the sheafification functor $\mathcal{S}$.

Corollary 3.21 (essential surjectivity) If the cover $\left\{U_{i}\right\}$ is p-good, then the sheafification functor $\mathcal{S}: \operatorname{Tw}_{\text {perf }}(X) \rightarrow \mathrm{Qcoh}_{\text {perf }}(X)$ induces an essentially surjective functor

$$
\mathcal{S}: \operatorname{HoTw}_{\text {perf }}(X) \rightarrow \mathrm{D}_{\text {perf }}(\mathrm{Q} \operatorname{coh}(X))
$$

Proof Let $P=\left(S^{\bullet}, d\right)$ be an object in $\operatorname{Qcoh}_{\text {perf }}(X)$. Consider the associated twisted complex $\mathcal{T}(P)$, by Proposition 3.20 there exist a twisted complex $\mathcal{E}$ together with a weak equivalence

$$
\phi: \mathcal{E} \stackrel{\sim}{\longrightarrow} \mathcal{T}(P)
$$

Then by Corollary 3.10 we get a quasi-isomorphism

$$
\mathcal{S}(\phi): \mathcal{S}(\mathcal{E}) \stackrel{\sim}{\longrightarrow} \mathcal{S} \mathcal{T}(P) .
$$

On the other hand, Proposition 3.12 provides us another quasi-isomorphism

$$
\tau_{P}: P \stackrel{\sim}{\longrightarrow} \mathcal{S} \mathcal{T}(P) .
$$

Therefore $\mathcal{S}(\mathcal{E})$ is quasi-isomorphic to $P$.

\subsubsection{Essential surjectivity on complexes of $\mathcal{O}_{X}$-modules}

Now we want to show that the functor $\mathcal{S}: \operatorname{HoTw}_{\text {perf }}(X) \rightarrow \mathrm{D}_{\text {perf }}(X)$ is essentially surjective. For this we need the following additional condition on the ringed space $\left(X, \mathcal{O}_{X}\right)$.

Definition 3.22 We say a locally ringed space $\left(X, \mathcal{O}_{X}\right)$ satisfies the perfect-equivalent condition if the natural map $\mathrm{D}_{\text {perf }}(\mathrm{Qcoh}(X)) \rightarrow \mathrm{D}_{\text {perf }}(X)$ is an equivalence.

Further discussions of perfect-equivalent condition will be given in Appendix 1. In particular, we can show that any quasi-compact and semi-separated scheme or any Noetherian scheme satisfies the perfect-equivalent condition.

Corollary 3.21 and Definition 3.22 imply

Corollary 3.23 (essential surjectivity) If $X$ satisfies the perfect-equivalent condition and the cover $\left\{U_{i}\right\}$ is p-good, then the functor

$$
\mathcal{S}: \operatorname{HoTw}_{\text {perf }}(X) \rightarrow \mathrm{D}_{\text {perf }}(X)
$$

is essentially surjective. 


\subsection{The fully-faithfulness of the sheafification functor}

\subsubsection{Fully faithful on complexes of quasi-coherent sheaves}

We want to show that the sheafification functor $\mathcal{S}$ induces a fully faithful functor $\mathcal{S}: \operatorname{HoTw}_{\text {perf }}(X) \rightarrow \mathrm{D}_{\text {perf }}(\mathrm{Q} \operatorname{coh}(X))$. First we have the following result.

Proposition 3.24 Let the cover $\left\{U_{i}\right\}$ satisfy $H^{k}\left(U_{i}, \mathcal{F}\right)=0$ for any $i$, any quasicoherent sheaf $\mathcal{F}$ on $U_{i}$ and any $k \geqslant 1$. If $\mathcal{E}$ and $\mathcal{F}$ are both in the subcategory $\operatorname{Tw}_{\text {perf }}(X)$, then $\mathcal{S}(\phi): \mathcal{S}(E) \rightarrow \mathcal{S}(\mathcal{F})$ is a quasi-isomorphism if and only if $\phi: \mathcal{E} \rightarrow \mathcal{F}$ is invertible in $\mathrm{HoTw}_{\text {perf }}(X)$.

Proof We first use Proposition 2.31, which claims that $\phi: \mathcal{E} \rightarrow \mathcal{F}$ is invertible in $\operatorname{HoTw}(X)$ if and only if $\phi$ is a weak equivalence. Moreover, Corollary 3.10 tells us $\phi$ is a weak equivalence if and only if $\mathcal{S}(\phi): \mathcal{S}(E) \rightarrow \mathcal{S}(\mathcal{F})$ is a quasi-isomorphism, hence we get the result.

Now we are about to prove the full-faithfulness of the functor $\mathcal{S}$. We divide the proof into several steps and first we have the following lemma.

Lemma 3.25 (fullness) If the cover $\left\{U_{i}\right\}$ is p-good, then the functor $\mathcal{S}: \operatorname{HoTw}_{\text {perf }}(X)$ $\rightarrow \mathrm{D}_{\text {perf }}(\mathrm{Q} \operatorname{coh}(X))$ is full.

Proof Let $\mathcal{A}$ and $\mathcal{B}$ be two objects in $\operatorname{Tw}_{\text {perf }}(X)$. A morphism $\mathcal{S}(\mathcal{A}) \rightarrow \mathcal{S}(\mathcal{B})$ in $\mathrm{D}_{\text {perf }}(\mathrm{Q} \operatorname{coh}(X))$ can be written as

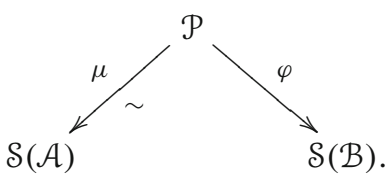

Applying $\mathcal{T}$ we get

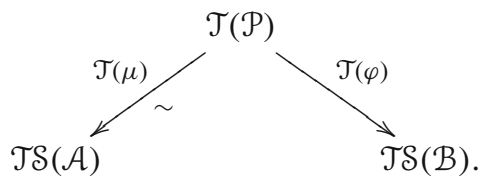

$\mathcal{P}$ is a perfect complex since it is quasi-isomorphic to $\mathcal{S}(\mathcal{A})$. Then by Proposition 3.20 there exists a resolution $\phi: \mathcal{E} \stackrel{\sim}{\longrightarrow} \mathcal{T}(\mathcal{P})$ and hence

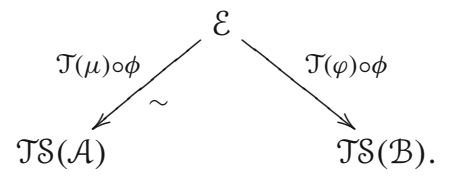


Compose with $\gamma_{\mathcal{A}}: \mathcal{T S}(\mathcal{A}) \rightarrow \mathcal{A}$ and $\gamma_{\mathcal{B}}: \mathcal{T S}(\mathcal{B}) \rightarrow \mathcal{B}$, we get

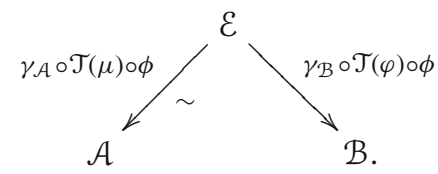

The left map $\gamma_{\mathcal{A}} \circ \mathcal{T}(\mu) \circ \phi$ is a weak equivalence between twisted perfect complexes hence by Proposition 2.31 it is invertible up to homotopy. Let $\theta: \mathcal{A} \rightarrow \mathcal{B}$ be the composition

$$
\theta=\gamma_{\mathcal{B}} \circ \mathcal{T}(\varphi) \circ \phi \circ\left(\gamma_{\mathcal{A}} \circ \mathcal{T}(\mu) \circ \phi\right)^{-1}
$$

It is clear that $\mathcal{S}(\theta)$ equals $\varphi \circ(\mu)^{-1}$ in the derived category. We know that the functor $\mathcal{S}$ is full.

Lemma 3.26 (faithfulness) If the cover $\left\{U_{i}\right\}$ is p-good, then $\mathcal{S}: \operatorname{HoTw}_{\text {perf }}(X) \rightarrow$ $\mathrm{D}_{\text {perf }}(\mathrm{Q} \operatorname{coh}(X))$ is faithful.

Proof Let $\theta: \mathcal{A} \rightarrow \mathcal{B}$ be a morphism between twisted perfect complexes such that $\mathcal{S}(\theta)=0$ in the derived category. Then, by definition, there is a complex $\mathcal{P}$ together with a quasi-isomorphism $\mu: \mathcal{P} \rightarrow \mathcal{S}(\mathcal{A})$ such that $\mathcal{S}(\theta) \circ \mu$ is homotopic to 0 . It follows that

$$
\mathcal{T}(\mathcal{P}) \stackrel{\mathcal{T}(\mu)}{\longrightarrow} \mathcal{T S}(\mathcal{A}) \stackrel{\mathcal{T}(\mathcal{S}(\theta))}{\longrightarrow} \mathcal{T S}(\mathcal{B})
$$

is homotopic to 0 .

On the other hand, we have the following commutative diagram:

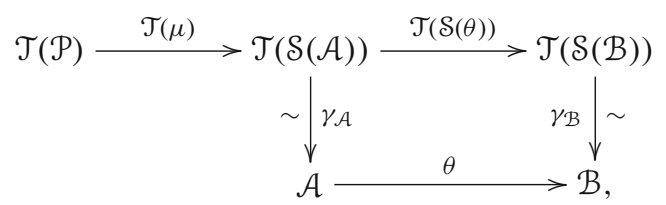

hence $\theta \circ \gamma_{\mathcal{A}} \circ \mathcal{T}(\mu)$ is homotopic to 0 and so is $\theta \circ \gamma_{\mathcal{A}} \circ \mathcal{T}(\mu) \circ \phi$, where $\phi: \mathcal{E} \rightarrow \mathcal{T}(\mathcal{P})$ is as in the proof of Lemma 3.25. From this we conclude that $\theta$ is homotopic to 0 because $\gamma_{\mathcal{A}} \circ \mathcal{T}(\mu) \circ \phi$ is invertible up to homotopy.

As an immediate corollary of Lemmas 3.25 and 3.26 we have

Corollary 3.27 If the cover $\left\{U_{i}\right\}$ is p-good, then $\mathcal{S}: \operatorname{HoTw}_{\text {perf }}(X) \rightarrow \mathrm{D}_{\text {perf }}(\mathrm{Qcoh}(X))$ is fully faithful.

Remark 3.28 The great advantage of twisted complexes is that we have more flexibility on morphisms. For example when $\left(X, \mathcal{O}_{X}\right)$ is a projective scheme, then it is well-known 
that any perfect complex on $X$ is strictly perfect. In other words, let $L(X)$ be the dgcategory of two-side bounded complexes of finitely generated locally free sheaves on $X$. Then the natural functor $\operatorname{HoL}(X) \rightarrow \mathrm{D}_{\text {perf }}(\mathrm{Q} \operatorname{coh}(X))$ is essentially surjective but not necessarily fully faithful.

In fact, let $\mathcal{E}$ and $\mathcal{F}$ be two objects in $\mathrm{L}(X)$ and $\phi: \mathcal{E} \stackrel{\sim}{\longrightarrow} \mathcal{F}$ be a quasi-isomorphism. Then in general $\phi$ does not have an inverse in $\operatorname{HoL}(X)$. Nevertheless the inverse of $\phi$ exists in $\operatorname{HoTw}_{\text {perf }}(X)$ if we consider $\mathcal{E}$ and $\mathcal{F}$ as twisted perfect complexes through the twisting functor $\mathcal{T}$ and the cover is p-good.

Now we can state the main theorem of this paper which is an immediate consequence of Corollaries 3.21 and 3.27.

Theorem 3.29 (dg-enhancement) If the cover $\left\{U_{i}\right\}$ is p-good, then the sheafification functor $\mathcal{S}: \mathrm{Tw}_{\text {perf }}(X) \rightarrow \mathrm{Qcoh}_{\mathrm{perf}}(X)$ gives an equivalence of categories

$$
\mathcal{S}: \operatorname{HoTw}_{\text {perf }}(X) \rightarrow \mathrm{D}_{\text {perf }}(\mathrm{Q} \operatorname{coh}(X)) \text {. }
$$

Example 3.30 We have the following cases when we can apply Theorem 3.29. In fact we only need to verify that the following spaces have p-good covers. For more discussion on p-good covers see Appendix 2.

- Let $\left(X, \mathcal{O}_{X}\right)$ be a separated scheme and $\left\{U_{i}\right\}$ be an affine cover, then we have an equivalence of categories $\mathcal{S}: \operatorname{HoTw}_{\text {perf }}(X) \rightarrow \mathrm{D}_{\text {perf }}(\mathrm{Q} \operatorname{coh}(X))$.

- Let $X$ be a complex manifold with the structure sheaf of holomorphic functions, then we have an equivalence of categories $\mathcal{S}: \operatorname{HoTw}_{\text {perf }}(X) \rightarrow \mathrm{D}_{\text {perf }}(\mathrm{Q} \operatorname{coh}(X))$.

- Let $X$ be a smooth manifold with the structure sheaf of smooth functions, then we have an equivalence of categories $\mathcal{S}: \operatorname{HoTw}_{\text {perf }}(X) \rightarrow \mathrm{D}_{\text {perf }}(\mathrm{Q} \operatorname{coh}(X))$.

\subsubsection{Fully faithful on complexes of $\mathcal{O}_{X}$-modules}

Similar to the discussion in Sect. 3.3.3, we can add certain conditions on $X$ and get the fully faithfulness on perfect complexes of arbitrary $\mathcal{O}_{X}$-modules. As a direct consequence of Corollary 3.27 and the perfect-equivalent condition (Definition 3.22) we have

Corollary 3.31 (fully faithful) If $X$ satisfies the perfect-equivalent condition and the cover $\left\{U_{i}\right\}$ is p-good, then the functor $\mathcal{S}: \operatorname{HoTw}_{\text {perf }}(X) \rightarrow \mathrm{D}_{\text {perf }}(X)$ is fully faithful.

Immediately from Corollaries 3.23 and 3.31 we have

Theorem 3.32 (dg-enhancement) If $X$ satisfies the perfect-equivalent condition and the cover $\left\{U_{i}\right\}$ is $p$-good, then the sheafification functor $\mathcal{S}: \operatorname{Tw}_{\text {perf }}(X) \rightarrow \operatorname{Sh}_{\text {perf }}(X)$ gives an equivalence of categories

$$
\mathcal{S}: \operatorname{HoTw}_{\text {perf }}(X) \rightarrow \mathrm{D}_{\text {perf }}(X)
$$

Example 3.33 The application of Theorem 3.32 is more restrictive than Theorem 3.29 since we need to verify the perfect-equivalent condition. Nevertheless it contains the following important cases: Let $\left(X, \mathcal{O}_{X}\right)$ be a quasi-compact and semi-separated or 
Noetherian scheme and $\left\{U_{i}\right\}$ be an affine cover, then we have an equivalence of categories $\mathcal{S}: \operatorname{HoTw}_{\text {perf }}(X) \rightarrow \mathrm{D}_{\text {perf }}(X)$. See Appendix 1, Corollary 6.6.

Remark 3.34 As we mentioned in Remark 1.2 in Introduction, twisted complexes are very similar to the Čech enhancement introduced in [12]. In fact for a complex of sheaves $\mathcal{E}$ on $X$, we could see that $\mathcal{S} \mathcal{T}(\mathcal{E})$ is almost the same as $\mathcal{E}_{\supset}$ in [12, Section 3.2.3]. Nevertheless, our twisted complexes and the Čech enhancement in [12] have the following two main differences:

- Twisted complexes allow twists $\left(a^{i, 1-i}\right)$ hence we could find resolutions of nonstrictly perfect complexes on non-GSP schemes.

- We do not have an order on the open subsets and we do not assume the open cover is finite.

- We do not use the pushforward $i$ ! hence we do not consider $\mathcal{E}_{\supset}$ as in $[12$, Section 3.2.3].

\section{Applications of twisted complexes}

Twisted complexes have various applications. For example in [15] twisted complexes are used to formulate and prove a Grothendieck-Riemann-Roch theorem for perfect complexes and in [9] they are used to compute the higher algebraic K-theory of schemes.

Remark 4.1 Neither of the above works uses the fact that twisted perfect complexes are a dg-enhancement of perfect complexes.

In this paper we talk about the application of twisted complexes in descent theory. It is well-known that one of the drawbacks of derived categories is that they do not satisfy descent. In more details, let $X$ be a scheme and $U, V$ be an open cover of $X$, then we have derived categories $\mathrm{D}_{\text {perf }}(X), \mathrm{D}_{\text {perf }}(U), \mathrm{D}_{\text {perf }}(V)$, and $\mathrm{D}_{\text {perf }}(U \cap V)$. Moreover, we have the fiber product of categories $\mathrm{D}_{\text {perf }}(U) \times \mathrm{D}_{\text {perf }}(U \cap V) \mathrm{D}_{\text {perf }}(V)$. However, the natural functor

$$
\mathrm{D}_{\text {perf }}(X) \rightarrow \mathrm{D}_{\text {perf }}(U) \times \mathrm{D}_{\text {perf }}(U \cap V) \mathrm{D}_{\text {perf }}(V)
$$

is not an equivalence even in the case that $X=\mathbb{P}^{1}$ and $U, V$ are the upper and lower hemispheres. See [18, Section 2.2(d)] for more details.

This problem can be solved in the framework of dg-categories. In fact, Tabuada in [16] gives an explicit construction of path object in dg-categories, which leads to the following definition of homotopy fiber product of dg-categories.

Definition 4.2 ([1, Section 4]) Let $A, B, C$ be dg-categories and $\phi: A \rightarrow C, \theta: B \rightarrow$ $C$ be dg-functors. Then the homotopy fiber product $A \times{ }_{C}^{h} B$ is a dg-category with objects

$$
\begin{aligned}
\mathrm{ob}\left(A \times_{C}^{h} B\right)=\{M, N, f: & M \in \mathrm{ob}(A), N \in \mathrm{ob}(B), \\
& f: \phi(M) \rightarrow \theta(N) \text { closed of degree } 0 \\
& \text { and invertible in } \left.H^{0}(C)\right\} .
\end{aligned}
$$


The degree $k$ morphisms between $\left(M_{1}, N_{1}, f_{1}\right)$ and $\left(M_{2}, N_{2}, f_{2}\right)$ are given by

$$
(\mu, v, \tau) \in A^{k}\left(M_{1}, M_{2}\right) \oplus B^{k}\left(N_{1}, N_{2}\right) \oplus C^{k-1}\left(\phi\left(M_{1}\right), \theta\left(N_{2}\right)\right)
$$

with composition given by

$$
\left(\mu^{\prime}, v^{\prime}, \tau^{\prime}\right)(\mu, v, \tau)=\left(\mu^{\prime} \mu, v^{\prime} v, \tau^{\prime} \phi(\mu)+\theta\left(v^{\prime}\right) \tau\right)
$$

The differential on the morphisms is given by

$$
d(\mu, v, \tau)=\left(d \mu, d \nu, d \tau+f_{2} \phi(\mu)-(-1)^{k} \theta(\nu) f_{1}\right) .
$$

Remark 4.3 We should mention that in [18, Section 5.3] Toën uses the injective enhancement $L_{p e}(X)$ and claims that

$$
L_{p e}(X) \stackrel{\sim}{\rightarrow} L_{p e}(U) \times_{L_{p e}(U \cap V)}^{h} L_{p e}(V)
$$

Moreover, in [1] the authors use the cohesive modules as another dg-enhancements and prove that they have the descent property.

Now we move on to the descent problem of twisted perfect complexes. Let $X$ be a separated scheme and $X=U \cup V$ be two open subsets. For simplicity let us consider the case that $U$ and $V$ are affine. Then $U \cap V$ is affine too. Moreover, $\{U, V\}$ gives an affine (hence p-good) open cover of $X$ and we have $\operatorname{Tw}_{\text {perf }}\left(X, \mathcal{O}_{X},\{U, V\}\right)$.

It is clear that $\operatorname{Tw}_{\text {perf }}\left(U, \mathcal{O}_{U},\{U\}\right)$ is exactly the dg-category of strictly perfect complexes on $U$. The same assertion holds for $\operatorname{Tw}_{\text {perf }}\left(V, \mathcal{O}_{V},\{V\}\right)$ and $\operatorname{Tw}_{\text {perf }}(U \cap$ $\left.V, \mathcal{O}_{U \cap V},\{U \cap V\}\right)$. There are natural dg-functors

$$
\phi: \operatorname{Tw}_{\text {perf }}\left(U, \mathcal{O}_{U},\{U\}\right) \rightarrow \operatorname{Tw}_{\text {perf }}\left(U \cap V, \mathcal{O}_{U \cap V},\{U \cap V\}\right)
$$

and

$$
\theta: \operatorname{Tw}_{\text {perf }}\left(V, \mathcal{O}_{V},\{V\}\right) \rightarrow \operatorname{Tw}_{\text {perf }}\left(U \cap V, \mathcal{O}_{U \cap V},\{U \cap V\}\right)
$$

given by restriction.

We omit the open covers and structure rings in the notation of the twisted perfect complexes and we have the following descent property.

Proposition 4.4 Let $X, U, V$ be as above, then we have a quasi-equivalence of $d g$ categories

$$
\operatorname{Tw}_{\text {perf }}(X) \stackrel{\sim}{\longrightarrow} \operatorname{Tw}_{\text {perf }}(U) \times{ }_{\operatorname{Tw}_{\text {perf }}(U \cap V)}^{h} \operatorname{Tw}_{\text {perf }}(V)
$$

Proof The main part of the proof is to untangle the definition of homotopy fiber product. Let $\mathcal{E}$ be an object in $\operatorname{Tw}_{\text {perf }}(X)$. Then it gives $E_{U}^{\bullet}$ on $U$ and $E_{V}^{\bullet}$ on $V$. 
It is clear that $E_{U}^{\bullet}$ together with $a_{U}^{0,1}$ give an object in $\operatorname{Tw}_{\text {perf }}(U)$ and we denote it by $\mathcal{M}$. Similarly $E_{V}^{\bullet}$ together with $a_{V}^{0,1}$ give an object $\mathcal{N}$ in $\operatorname{Tw}_{\text {perf }}(V)$. Moreover, the map $a_{V U}^{1,0}$ gives the morphism $f: \mathcal{M} \rightarrow \mathcal{N}$ and it is homotopic invertible since $a$ satisfies the Maurer-Cartan equation and has the non-degenerate property.

Hence we get a dg-functor

$$
R: \operatorname{Tw}_{\text {perf }}(X) \rightarrow \operatorname{Tw}_{\text {perf }}(U) \times{ }_{\operatorname{Tw}_{\text {perf }}(U \cap V)}^{h} \operatorname{Tw}_{\text {perf }}(V) .
$$

It is clear that $R$ is essentially surjective. By the same method as in the proof of Proposition 3.20 we can prove it is also quasi-fully faithful.

Remark 4.5 The same idea works for the general case where $U$ and $V$ are not affine. Nevertheless we need an explicit construction of homotopy limit of dg-categories and this topic will be treated in another paper.

\section{Further topics}

\subsection{Twisted coherent complexes}

In this subsection we consider a variation of twisted perfect complexes, where the two-side bounded complexes are replaced by bounded above complexes. We omit most of the proofs since they are the same as the corresponding proofs for the twisted perfect complexes.

\subsubsection{The derived category of bounded above coherent complexes}

First we review the relevant derived categories. We have a definition of coherent complex.

Definition 5.1 Let $\left(X, \mathcal{O}_{X}\right)$ be a separated, Noetherian scheme. A complex $\mathcal{S}^{\bullet}$ of $\mathcal{O}_{X}$-modules is bounded above and coherent if for any point $x \in X$, there exist an open neighborhood $U$ of $x$ and a bounded above complex of finite rank, locally free sheaves $\mathcal{E}_{U}^{\bullet}$ on $U$ such that the restriction $\left.\mathcal{S}^{\bullet}\right|_{U}$ is isomorphic to $\mathcal{E}_{U}^{\bullet}$ in $D\left(\left.\mathcal{O}_{X}\right|_{U}\right.$-mod), the derived category of sheaves of $\mathcal{O}_{X}$-modules on $U$.

Remark 5.2 If $X$ is not a separated Noetherian scheme then the category of bounded above coherent complexes does not behave well. In fact a more standard notion is the pseudo-coherent complex on a ringed space, see [2, Exposé I] or [17, Section 2]. Nevertheless, pseudo-coherent coincides with our definition of coherent if $X$ is a Noetherian scheme as shown in Appendix 1. In this paper we will stick to the above definition of coherent complex.

In this subsection we always assume $X$ is a separated Noetherian scheme. We consider the following categories.

Definition 5.3 Let $\mathrm{Sh}_{\text {coh }}^{-}(X)$ be the full dg-subcategory of $\operatorname{Sh}(X)$ which consists of bounded above coherent complexes on $X$. Similarly we have $\mathrm{K}_{\mathrm{coh}}^{-}(X), \mathrm{D}_{\mathrm{coh}}^{-}(X)$, $\mathrm{K}_{\mathrm{coh}}^{-}(\mathrm{Q} \operatorname{coh}(X))$, and $\mathrm{D}_{\text {coh }}^{-}(\mathrm{Q} \operatorname{coh}(X))$. 


\subsubsection{Twisted coherent complexes}

We have the following definition which is similar to Definition 2.14.

Definition 5.4 A twisted coherent complex $\mathcal{E}=\left(E^{\bullet}, a\right)$ is the same as twisted complex except that $E^{\bullet}$ are bounded above graded finitely generated locally free $\mathcal{O}_{X}$-modules. The twisted coherent complexes form a dg-category and we denote it by $\mathrm{Tw}_{\text {coh }}^{-}\left(X, \mathcal{O}_{X},\left\{U_{i}\right\}\right)$ or simply $\mathrm{Tw}_{\text {coh }}^{-}(X)$. Obviously $\mathrm{Tw}_{\text {coh }}^{-}(X)$ is a full $\mathrm{dg}$ subcategory of $\operatorname{Tw}(X)$ while $\operatorname{Tw}_{\text {perf }}(X)$ is a full dg-subcategory of $\operatorname{Tw}_{\text {coh }}^{-}(X)$.

The differential $\delta_{a}$, shift functor, mapping cone and weak equivalence as in Sects. 2.5 and 2.6 can be defined on $\mathrm{Tw}_{\mathrm{coh}}^{-}(X)$ without any change. Moreover we have the same result as in Proposition 2.31.

Proposition 5.5 Let the cover $\left\{U_{i}\right\}$ satisfy $H^{k}\left(U_{i}, \mathcal{F}\right)=0$ for any $i$, any quasicoherent sheaf $\mathcal{F}$ on $U_{i}$ and any $k \geqslant 0$. If $\mathcal{E}$ and $\mathcal{F}$ are both in the subcategory $\operatorname{Tw}_{\mathrm{coh}}^{-}(X)$, then a closed degree zero morphism $\phi$ between twisted complexes $\mathcal{E}$ and $\mathcal{F}$ is a weak equivalence if and only if $\phi$ is invertible in the homotopy category $\operatorname{HoTw}_{\mathrm{coh}}(X)$.

Proof Notice that in the proof of Proposition 2.31 we do not use the boundedness of the complexes hence the same proof works for $\operatorname{HoTw}_{\mathrm{coh}}(X)$.

\subsubsection{The sheafification functor on twisted coherent complexes}

We wish to restrict the sheafification functor in Definition 3.3 to twisted coherent complexes and get a dg-functor $\mathcal{S}: \operatorname{Tw}_{\mathrm{coh}}^{-}(X) \rightarrow \operatorname{Sh}(X)$ and we want to achieve a result which is similar to Remark 3.4, i.e. we want the dg-functor $\mathcal{S}$ maps $\operatorname{Tw}_{\mathrm{coh}}^{-}(X)$ to complexes of quasi-coherent sheaves. However, there is a serious problem here. Recall that in (13) of the definition of $\mathcal{S}$ we have

$$
\mathcal{S}^{n}(\mathcal{E})=\left.\prod_{p+q=n} E_{i_{0}}^{q}\right|_{U_{i_{0} \ldots i_{p}}} .
$$

Now $\mathcal{E}=\left(E_{i}^{\bullet}, a\right)$ is a twisted coherent complex hence $E_{i}^{\bullet}$ is bounded above. Therefore, $\left.\prod_{p+q=n} E_{i_{0}}^{q}\right|_{U_{i_{0} \ldots i p}}$ is an infinite product. The problem is that the category $\operatorname{Qcoh}(X)$ does not have infinite direct products for general $X$, and even when it has, the infinite direct product in $\mathrm{Q} c o h(X)$ is not the same as the product in the larger category $\operatorname{Sh}(X)$.

To solve this problem we have to slightly modify the definition of $\mathcal{S}$. First we introduce the following definition.

Definition 5.6 A twisted quasi-coherent complex $\mathcal{E}=\left(E^{\bullet}, a\right)$ is the same as twisted complex except that $E^{\bullet}$ are graded quasi-coherent $\mathcal{O}_{X}$-modules. The twisted quasicoherent complexes form a dg-category and we denote it by $\operatorname{Tw}_{\mathrm{qcoh}}\left(X, \mathcal{O}_{X},\left\{U_{i}\right\}\right)$ or simply $\operatorname{Tw}_{\mathrm{qcoh}}(X)$. Obviously, $\operatorname{Tw}_{\mathrm{qcoh}}(X)$ is a full dg-subcategory of $\operatorname{Tw}(X)$ while $\operatorname{Tw}_{\text {perf }}(X)$ and $\mathrm{Tw}_{\text {coh }}^{-}(X)$ are full dg-subcategories of $\operatorname{Tw}_{\mathrm{qcoh}}(X)$.

Before defining the sheafification functor we need the following lemma.

Lemma 5.7 ([17, Lemma B.12]) Let $X$ be a quasi-compact and quasi-separated scheme, then the category $\mathrm{Qcoh}(X)$ has all limits. 
Then we define the sheafification functor in a slightly modified way and we will call it $\widetilde{\mathcal{S}}$.

Definition 5.8 Let $X$ be a separated Noetherian (hence quasi-compact and quasiseparated) scheme. The definition of $\widetilde{\mathcal{S}}: \operatorname{Tw}_{\mathrm{qcoh}}(X) \rightarrow \mathrm{Q} \operatorname{coh}(X)$ is the same as that of $\mathcal{S}$ in Definition 3.3 except that in the equation

$$
\widetilde{\mathcal{S}}^{n}(\mathcal{E})=\left.\prod_{p+q=n} E_{i_{0}}^{q}\right|_{U_{i_{0} \ldots i p}}
$$

we take the direct product in Qcoh $(X)$. By Lemma 5.7, $\widetilde{\mathcal{S}}$ is well-defined.

Remark 5.9 The functor $\widetilde{\mathcal{S}}$ coincides with $\mathcal{S}$ if restricted to $\operatorname{Tw}_{\text {perf }}(X)$ since in this case the product $\left.\prod_{p+q=n} E_{i_{0}}^{q}\right|_{U_{i_{0} \ldots i p}}$ is finite and the product in Qcoh $(X)$ coincides with that in $\operatorname{Sh}(X)$.

Keep in mind that Proposition 3.5 works for any twisted complexes, hence it works for twisted coherent complexes. Moreover, following similar arguments, we also have the same result as in Corollary 3.8.

Proposition 5.10 If $\mathcal{E}=\left(E^{\bullet}, a\right)$ is a twisted coherent complex, then the sheafification $\widetilde{\mathcal{S}} \bullet(\mathcal{E})$ is a coherent complex of sheaves on $\left(X, \mathcal{O}_{X}\right)$. In other words, the sheafification functor $\widetilde{\mathcal{S}}$ restricts to $\mathrm{Tw}_{\mathrm{coh}}^{-}(X)$ and gives the following dg-functor:

$$
\widetilde{\mathcal{S}}: \mathrm{Tw}_{\mathrm{coh}}^{-}(X) \rightarrow \mathrm{Qcoh}_{\mathrm{coh}}^{-}(X) .
$$

\subsubsection{The essential surjectivity in the coherent case}

Similar to the discussion in Sect. 3.3, the dg-functor $\widetilde{\mathcal{S}}: \mathrm{Tw}_{\mathrm{coh}}^{-}(X) \rightarrow \mathrm{Qcoh}_{\mathrm{coh}}^{-}(X)$ induces a functor $\widetilde{\mathcal{S}}: \mathrm{HoTw}_{\mathrm{coh}}^{-}(X) \rightarrow \mathrm{D}_{\text {coh }}^{-}(\mathrm{Q} \operatorname{coh}(X))$. In this subsection we will show that this functor is essentially surjective under some mild condition. Moreover we will show that the functor $\widetilde{\mathcal{S}}: \operatorname{HoTw}_{\text {coh }}^{-}(X) \rightarrow \mathrm{D}_{\text {coh }}^{-}(X)$ is essentially surjective under some additional conditions.

First we have the following definitions which are similar to Definitions 3.16 and 3.18 .

Definition 5.11 A locally ringed space $\left(U, \mathcal{O}_{U}\right)$ is called $c$-good if it satisfies:

- For every coherent complex $\mathcal{C}^{\bullet}$ on $U$ which consists of quasi-coherent sheaves, there exist a bounded above complex of finitely generated locally free sheaves $\mathcal{E}^{\bullet}$ together with a quasi-isomorphism $v: \mathcal{E}^{\bullet} \stackrel{\sim}{\longrightarrow} \mathrm{C}^{\bullet}$.

- The higher cohomologies of quasi-coherent sheaves vanish, i.e. $H^{k}(U, \mathcal{F})=0$ for any quasi-coherent sheaf $\mathcal{F}$ on $U$ and any $k \geqslant 1$.

Remark 5.12 The letter "c" in the term "c-good space" stands for "coherent".

Definition 5.13 Let $\left(X, \mathcal{O}_{X}\right)$ be a locally ringed space, an open cover $\left\{U_{i}\right\}$ of $X$ is called a $c$-good cover if $\left(U_{I},\left.\mathcal{O}_{X}\right|_{U_{I}}\right)$ is a c-good space for any finite intersection $U_{I}$ of the open cover. 
For a separated, Noetherian scheme $\left(X, \mathcal{O}_{X}\right)$, any affine cover $\left\{U_{i}\right\}$ is c-good, see Appendix 2.

Then we have the coherent version of twisted resolution, the proof is similar to the one of Proposition 3.20.

Proposition 5.14 Assume the cover $\left\{U_{i}\right\}$ is c-good. Let $P=\left(S^{\bullet}, d_{S}\right)$ be a bounded above coherent complex which consists of quasi-coherent modules, then there exist a twisted coherent complex $\mathcal{E}$ together with a weak equivalence

$$
\phi: \mathcal{E} \stackrel{\sim}{\longrightarrow} \mathcal{T}(P)
$$

Hence we have the following essential surjectivity, the proof is similar to the one of Corollary 3.21 .

Corollary 5.15 If the cover $\left\{U_{i}\right\}$ is c-good, then $\widetilde{\mathcal{S}}: \mathrm{Tw}_{\mathrm{coh}}^{-}(X) \rightarrow \mathrm{Qcoh}_{\mathrm{coh}}^{-}(X)$ induces an essentially surjective functor $\widetilde{S}: \operatorname{HoTw}_{\text {coh }}^{-}(X) \rightarrow \mathrm{D}_{\text {coh }}^{-}(\mathrm{Q} \operatorname{coh}(X))$.

The essential surjectivity on arbitrary $\mathcal{O}_{X}$-modules involves the following definition.

Definition 5.16 We say a locally ringed space $\left(X, \mathcal{O}_{X}\right)$ satisfies the coherentequivalent condition if the natural map $\mathrm{D}_{\text {coh }}^{-}(\mathrm{Q} \operatorname{coh}(X)) \rightarrow \mathrm{D}_{\text {coh }}^{-}(X)$ is an equivalence.

Actually we can show that any Noetherian scheme with finite Krull dimension satisfies the coherent-equivalent condition, see Appendix 1, Corollary 6.8.

Corollary 5.15 and Definition 5.16 imply

Corollary 5.17 If $X$ satisfies the coherent-equivalent condition and the cover $\left\{U_{i}\right\}$ is $c$-good, then the functor $\widetilde{\mathcal{S}}: \mathrm{HoTw}_{\mathrm{coh}}^{-}(X) \rightarrow \mathrm{D}_{\mathrm{coh}}^{-}(X)$ is essentially surjective.

\subsubsection{The fully-faithfulness on coherent complexes}

Due to Proposition 5.5, following the proof of Proposition 3.24, we obtain

Proposition 5.18 Let the cover $\left\{U_{i}\right\}$ satisfy $H^{k}\left(U_{i},\left.\mathcal{O}_{X}\right|_{U_{i}}\right) \equiv 0$ for any $i$ and any $k \geqslant 0$. If $\mathcal{E}$ and $\mathcal{F}$ are both in the subcategory $\operatorname{Tw}_{\mathrm{coh}}^{-}(X)$, then $\widetilde{\mathcal{S}}(\phi): \widetilde{\mathcal{S}}(E) \rightarrow \widetilde{\mathcal{S}}(\mathcal{F})$ is a quasi-isomorphism if and only if $\phi: \mathcal{E} \rightarrow \mathcal{F}$ is invertible in $\operatorname{HoTw}_{\mathrm{coh}}^{-}(X)$.

Analogously to Corollary 3.27 , we have

Corollary 5.19 If the cover $\left\{U_{i}\right\}$ is c-good, then $\widetilde{\mathcal{S}}: \operatorname{HoTw}_{\text {coh }}^{-}(X) \rightarrow \mathrm{D}_{\text {coh }}^{-}(\operatorname{Qcoh}(X))$ is fully faithful.

Further, as an immediate consequence of Corollaries 5.15 and 5.19, we have

Theorem 5.20 If the cover $\left\{U_{i}\right\}$ is c-good, then the sheafification functor $\widetilde{\mathcal{S}}: \operatorname{Tw}_{\mathrm{coh}}^{-}(X)$ $\rightarrow \mathrm{Qcoh}_{\mathrm{coh}}^{-}(X)$ gives an equivalence of categories

$$
\widetilde{\mathcal{S}}: \operatorname{HoTw}_{\mathrm{coh}}^{-}(X) \rightarrow \mathrm{D}_{\mathrm{coh}}^{-}(\mathrm{Q} \operatorname{coh}(X))
$$

Example 5.21 If $X$ is a separated Noetherian scheme and $\left\{U_{i}\right\}$ is an affine cover, then we have an equivalence of categories $\widetilde{\mathcal{S}}: \operatorname{HoTw}_{\text {coh }}^{-}(X) \rightarrow \mathrm{D}_{\text {coh }}^{-}(\mathrm{Q} \operatorname{coh}(X))$. 
Now consider the coherent complexes of arbitrary $\mathcal{O}_{X}$-modules. As an immediate consequence of Theorem 5.20 and Definition 5.16, we have

Theorem 5.22 If $X$ satisfies the coherent-equivalent condition and the cover $\left\{U_{i}\right\}$ is $c$-good, then the sheafification functor $\widetilde{\mathcal{S}}: \mathrm{Tw}_{\mathrm{coh}}^{-}(X) \rightarrow \mathrm{Qcoh}_{\mathrm{coh}}^{-}(X)$ gives an equivalence of categories

$$
\widetilde{\mathcal{S}}: \operatorname{HoTw}_{\mathrm{coh}}^{-}(X) \rightarrow \mathrm{D}_{\mathrm{coh}}^{-}(X)
$$

Example 5.23 If $X$ is a separated Noetherian scheme with finite Krull dimension and $\left\{U_{i}\right\}$ is an affine cover, then we have an equivalence of categories $\widetilde{\mathcal{S}}: \operatorname{HoTw}_{\text {coh }}^{-}(X) \rightarrow$ $\mathrm{D}_{\text {coh }}^{-}(X)$. See Appendix 1, Corollary 6.8.

\subsection{Degenerate twisted complexes}

Recall that in the definition of twisted complex we have the non-degenerate condition which requires that on each $U_{i}$ we have

$$
a_{i i}^{1,0}=\mathrm{id}
$$

up to homotopy. It is interesting to see what happens if we drop the non-degenerate condition. In fact we have the following definition.

Definition 5.24 A generalized twisted complex is the same as a twisted complex except that we do not require $a_{i i}^{1,0}=$ id up to homotopy. Similarly we have generalized twisted perfect complexes and generalized twisted coherent complexes. We denote the dg-category of generalized twisted complexes by $\operatorname{gTw}(X)$. Similarly we have $\mathrm{gTw}_{\text {perf }}(X)$ and $\mathrm{gTw}_{\text {coh }}^{-}(X)$.

Example 5.25 For given $E_{i}^{\bullet}$, we could set all $a^{k, 1-k}$ to be 0 . It definitely satisfies the Maurer-Cartan equation $\delta a+a \cdot a=0$ hence it gives a generalized twisted complex but not a twisted complex unless $E_{i}^{\bullet}$ are all zero.

For generalized twisted complexes we have the following obvious observations:

- $\operatorname{Tw}(X)$ is a full dg-subcategory of $\operatorname{gTw}(X), \operatorname{Tw}_{\text {perf }}(X)$ is a full dg-subcategory of $\mathrm{gTw}_{\text {perf }}(X)$ and $\mathrm{Tw}_{\text {coh }}^{-}(X)$ is a full dg-subcategory of $\mathrm{gTw}_{\mathrm{coh}}^{-}(X)$.

- Nevertheless there is no inclusion relation between $\operatorname{gTw}_{\text {perf }}(X)$ and $\operatorname{Tw}(X)$ nor between $\mathrm{gTw}_{\mathrm{coh}}^{-}(X)$ and $\operatorname{Tw}(X)$.

- The pre-triangulated structure as in $\operatorname{Sect} 2.5$ can be defined on $g \operatorname{Tw}(X), \mathrm{gTw}_{\text {perf }}(X)$ and $\mathrm{gTw}_{\mathrm{coh}}^{-}(X)$ without any change.

- The weak equivalence in $\operatorname{gTw}(X)$ is exactly the same as in Sect. 2.6. Moreover, Definition 2.27 and Proposition 2.31 still hold for generalized twisted complexes.

- We can define the sheafification functor $S: \operatorname{gTw}(X) \rightarrow \operatorname{Sh}(X)$ in the same way as in Definitions 3.1 and 3.3. 
It is not obvious that $S$ maps a generalized twisted perfect/coherent complex to a perfect/coherent complex. Actually we need some more work. Recall, Lemma 2.19 claims that if the maps $a^{k, 1-k}$ satisfy the Maurer-Cartan equation, then $a_{i i}^{1,0}:\left(E_{i}^{n}, a_{i}^{0,1}\right) \rightarrow$ $\left(E_{i}^{n}, a_{i}^{0,1}\right)$ is an idempotent map in the homotopy category $\mathrm{K}\left(U_{i}\right)$, i.e. $\left(a_{i i}^{1,0}\right)^{2}=a_{i i}^{1,0}$ up to chain homotopy.

It is a classical result that the category $\mathrm{K}\left(U_{i}\right)$ is idempotent complete [6, Proposition 3.2], i.e. for any object $S$ of $\mathrm{K}\left(U_{i}\right)$ and any idempotent $\alpha: S \rightarrow S$, there exists a splitting of $\alpha$. More precisely, there exist a splitting $T$ in $\mathrm{K}\left(U_{i}\right)$ together with $i: T \rightarrow S$ and $p: S \rightarrow T$ such that

$$
p i=\mathrm{id}_{T}, \quad i p=\alpha .
$$

Intuitively such splitting $T$ can be considered as the image of the map $\alpha$. However, in general $T$ is not the naïve image of $\alpha$ in the chain complex.

The following proposition gives an explicit construction of the splitting.

Proposition 5.26 Let $\mathcal{E}=\left(E_{i}^{\bullet}, a\right)$ be a generalized twisted complex and $\left(\mathcal{S}^{\bullet}(\mathcal{E}), \delta_{a}\right)$ be the associated complex of sheaves. Then $\left.\left(\mathcal{S}^{\bullet}(\mathcal{E}), \delta_{a}\right)\right|_{U_{j}}$ is a splitting of the idempotent $a_{j j}^{1,0}:\left(E_{j}^{n}, a_{j}^{0,1}\right) \rightarrow\left(E_{j}^{n}, a_{j}^{0,1}\right)$, i.e. we have two morphisms

$$
f:\left.\left(\mathcal{S}^{\bullet}(\mathcal{E}), \delta_{a}\right)\right|_{U_{j}} \rightarrow\left(E_{j}^{\bullet}, a_{j}^{0,1}\right), \quad g:\left.\left(E_{j}^{\bullet}, a_{j}^{0,1}\right) \rightarrow\left(\mathcal{S}^{\bullet}(\mathcal{E}), \delta_{a}\right)\right|_{U_{j}}
$$

such that

$$
f \circ g=a_{j j}^{1,0}, \quad g \circ f=\left.\operatorname{id}_{\mathcal{S}} \bullet(\mathcal{E})\right|_{U_{j}} \quad \text { up to chain homotopy. }
$$

Proof The proof is exactly the same as that of Proposition 3.5 except that here $f \circ g=$ $a_{j j}^{1,0}$ is not necessarily equal to id, not even up to homotopy.

With the help of Proposition 5.26 we can get the following result.

Corollary 5.27 If $\mathcal{E}=\left(E^{\bullet}, a\right)$ is a generalized twisted perfect (or twisted coherent) complex, then the sheafification $\mathcal{S}^{\bullet}(\mathcal{E})$ is a perfect (or coherent, respectively) complex of sheaves on $\left(X, \mathcal{O}_{X}\right)$. In other words, the sheafification functor $\mathcal{S}$ restricts to $\mathrm{gTw}_{\text {perf }}(X)$ (or $\mathrm{gTw}_{\mathrm{coh}}^{-}(X)$, respectively) and gives the following dg-functor:

$$
\mathcal{S}: \mathrm{gTw}_{\mathrm{perf}}(X) \rightarrow \mathrm{Qcoh}_{\mathrm{perf}}(X), \quad \mathcal{S}: \mathrm{gTw}_{\mathrm{coh}}^{-}(X) \rightarrow \mathrm{Qcoh}_{\mathrm{coh}}(X)
$$

Proof Since $\mathcal{E}=\left(E^{\bullet}, a\right)$ is a generalized twisted perfect complex, for each $U_{j}$ the complex $\left(E_{j}^{\bullet}, a_{j}^{0,1}\right)$ is a two-side bounded complex which consists of locally free finitely generated $\mathcal{O}_{X}$-modules, i.e. $\left(E_{j}^{\bullet}, a_{j}^{0,1}\right)$ is an object in $\mathrm{K}_{\text {perf }}\left(U_{j}\right)$. We know that $\mathrm{K}_{\text {perf }}\left(U_{j}\right)$ is also idempotent complete since it consists of compact objects in $\mathrm{K}\left(U_{j}\right)$. Proposition 5.26 tells us that $\left.\mathcal{S}^{\bullet}(\mathcal{E})\right|_{U_{j}}$ is a splitting of idempotent $a_{j j}^{1,0}$ hence $\left.\mathcal{S}^{\bullet}(\mathcal{E})\right|_{U_{j}}$ is perfect on $U_{j}$. Moreover, this is true for any member $U_{j}$ of the open cover, therefore $\mathcal{S}^{\bullet}(\mathcal{E})$ is a perfect complex of sheaves on $\left(X, \mathcal{O}_{X}\right)$. The same proof works for twisted coherent complexes. 
Corollary 5.28 - If the cover $\left\{U_{i}\right\}$ is p-good, then the functor

$$
\mathcal{S}: \operatorname{Ho}\left(\mathrm{gTw}_{\text {perf }}(X)\right) \rightarrow \mathrm{D}_{\text {perf }}(\mathrm{Q} \operatorname{coh}(X))
$$

is essentially surjective.

- If the cover $\left\{U_{i}\right\}$ is c-good, then the functor

$$
\mathcal{S}: \operatorname{Ho}\left(\mathrm{gTw}_{\mathrm{coh}}^{-}(X)\right) \rightarrow \mathrm{D}_{\mathrm{coh}}(\mathrm{Q} \operatorname{coh}(X))
$$

is essentially surjective.

Proof By Corollary 3.21 we already know that $\mathcal{S}: \operatorname{HoTw}_{\text {perf }}(X) \rightarrow \mathrm{D}_{\text {perf }}(\mathrm{Qcoh}(X))$ is essentially surjective. Since $\operatorname{Tw}_{\text {perf }}(X)$ is a subcategory of $\mathrm{gTw}_{\text {perf }}(X)$ and the functors $\mathcal{S}$ coincide on $\operatorname{Tw}_{\text {perf }}(X)$, the claim is obviously true. The same proof works for twisted coherent complexes.

However, $\mathcal{S}$ does not induce a fully faithful functor

$$
\mathcal{S}: \operatorname{Ho}\left(\mathrm{gTw}_{\text {perf }}(X)\right) \rightarrow \mathrm{D}_{\text {perf }}(\mathrm{Q} \operatorname{coh}(X))
$$

nor

$$
\mathcal{S}: \operatorname{Ho}\left(\mathrm{gTw}_{\mathrm{coh}}^{-}(X)\right) \rightarrow \mathrm{D}_{\mathrm{coh}}(\mathrm{Q} \operatorname{coh}(X))
$$

The main reason of the failure is that we no longer have the same result as in Corollary 3.10 for generalized twisted complexes and Proposition 2.31 does not hold for generalized twisted complexes either.

In fact, if $\mathcal{E}$ and $\mathcal{F}$ are generalized twisted coherent complexes, then the fact that $\mathcal{S}(\phi): \mathcal{S}(\mathcal{E}) \rightarrow \mathcal{S}(\mathcal{F})$ is a quasi-isomorphism does not imply $\phi: \mathcal{E} \rightarrow \mathcal{F}$ is invertible in the homotopy category.

Example 5.29 For a counter-example, let $\mathcal{E}=\left(E_{i}^{\bullet}, 0\right)$ be non-zero, two-side bounded graded locally free finitely generated $\mathcal{O}_{X}$-modules on each $U_{i}$ with all $a$ equal to 0 . Let $\mathcal{F}$ simply be 0 and $\phi$ be the zero map. It is clear that $\phi_{i}^{0,0}: E_{i}^{\bullet} \rightarrow 0$ is not a quasi-isomorphism hence $\phi$ cannot be invertible in $\mathrm{Ho}\left(\mathrm{gTw}_{\text {perf }}(X)\right)$. However, by Proposition 5.26 it is not difficult to show that $\mathcal{S}(\mathcal{E})$ is an acyclic complex hence $\mathcal{S}(\phi)=0: \mathcal{S}(\mathcal{E}) \rightarrow 0$ is a quasi-isomorphism.

The above discussion tells us that $\left(\mathrm{gTw}_{\text {perf }}(X), \mathcal{S}\right)$ (or $\left.\left(\mathrm{gTw}_{\text {coh }}^{-}(X), \mathcal{S}\right)\right)$ is not a dg-enhancement of $\mathrm{D}_{\text {perf }}(\mathrm{Q} \operatorname{coh}(X))$ (or $\mathrm{D}_{\text {coh }}(\mathrm{Q} \operatorname{coh}(X))$ respectively). Nevertheless, $\mathrm{gTw}(X)$ has its own interests and may be further studied in the future.

\subsection{Quillen adjunction}

The proof of dg-enhancement in this paper is more or less a by-hand proof. Nevertheless in this section we would like to briefly mention a more categorical approach which we hope can give a systematic proof of the result in this paper. 
We have defined two dg-functors $\mathcal{S}: \operatorname{Tw}(X) \rightarrow \operatorname{Sh}(X)$ and $\mathcal{T}: \operatorname{Sh}(X) \rightarrow \operatorname{Tw}(X)$. We have found the relations between them in Propositions 3.12, 3.13 and 3.15.

On the other hand, we have the injective and projective model structure on $\mathrm{Sh}(X)$, see [11]. Moreover, in Definition 2.27 we already have a notion of weak equivalence in $\operatorname{Tw}(X)$ and we wish to further construct a suitable model structure on $\operatorname{Tw}(X)$ with the weak equivalence as above, which, together with the suitable model structure on $\operatorname{Sh}(X)$, makes $\mathcal{S}$ and $\mathcal{T}$ a Quillen adjunction and further a Quillen equivalence

$$
\mathcal{S}: \operatorname{Tw}(X) \leftrightarrows \operatorname{Sh}(X): \mathcal{T}
$$

The Quillen adjunction, if exists, will reveal deeper information on twisted complexes. It is also hoped that the dg-enhancement result can be proved in this approach.

\section{Appendix 1: Some discussions on complexes of sheaves}

\section{Pseudo-coherent complexes and coherent complexes}

Recall that we have Definition 5.1 of coherent complexes in Sect. 5.1. For general locally ringed spaces $\left(X, \mathcal{O}_{X}\right)$, this version of coherent complex does not behave well and we have the following definition.

Definition 6.1 ([17, Definitions 2.1.1 and 2.2.6] or [2, Exposé I,2.1,2.3])

- For an integer $n$, a complex $\mathcal{E}^{\bullet}$ of $\mathcal{O}_{X}$-modules on $X$ is called strictly $n$-pseudocoherent if $\mathcal{E}^{i}$ is a locally free finitely generated $\mathcal{O}_{X}$-module for $i \geqslant n$ and $\mathcal{E}^{i}=0$ for $i$ sufficiently large.

- A complex $\mathcal{E}^{\bullet}$ of $\mathcal{O}_{X}$-modules on $X$ is called strictly pseudo-coherent if it is $n$ strictly-pseudo-coherent for all $n$, i.e. it is a bounded above complex of locally free finitely generated $\mathcal{O}_{X}$-modules.

- For any integer $n$, a complex $\mathcal{E}^{\bullet}$ of $\mathcal{O}_{X}$-modules on $X$ is called m-pseudo-coherent if for any point $x \in X$ there exist an open neighborhood $x \in U \subset X$ and a morphism of complexes $\alpha:\left.\mathcal{P}_{U}^{\bullet} \rightarrow \mathcal{E}^{\bullet}\right|_{U}$ where $\mathcal{P}_{U}$ is strictly $n$-pseudo-coherent on $U$ and $\alpha$ is a quasi-isomorphism on $U$.

- We say $\mathcal{E}^{\bullet}$ is pseudo-coherent if it is $n$-pseudo-coherent for all $n$.

We may hope that a pseudo-coherent complex is locally quasi-isomorphic to a strictly pseudo-coherent complex. However according to [17, 2.2.7] it is not true in general:

For a pseudo-coherent complex of general $\mathcal{O}_{X}$-modules, there will locally be $n$-quasi-isomorphisms with a strictly pseudo-coherent complex, but the local neighborhoods where the $n$-quasi-isomorphisms are defined may shrink as $n$ goes to $-\infty$, and so may fail to exist in the limit. So there may not be a local quasi-isomorphism with a strict pseudo-coherent complex.

As a result, the definition of pseudo-coherent complex and our definition of coherent complex are not equivalent in general. Nevertheless if we assume $X$ is a Noetherian scheme, then we have the following proposition. 
Proposition 6.2 ([17, 2.2.8], [2, Exposé I, Section 3]) A complex $E^{\bullet}$ of $\mathcal{O}_{X}$-modules on a Noetherian scheme $X$ is pseudo-coherent if and only if $E^{\bullet}$ is cohomologically bounded above and all $H^{k}\left(E^{\bullet}\right)$ are coherent $\mathcal{O}_{X}$-modules, i.e. $E^{\bullet}$ is pseudo-coherent if and only if $E^{\bullet} \in \mathrm{D}_{\text {coh }}^{-}(X)$.

\section{Quasi-coherent modules v.s. arbitrary $\mathcal{O}_{X}$-modules}

It is a subtle but important question whether we could replace a complex of $\mathcal{O}_{X^{-}}$ modules by a complex of quasi-coherent modules in the derived categories. In this subsection we collect some results on this topic which can be found in [17, Appendix B] and [2, Exposé II].

Definition 6.3 Let $\left(X, \mathcal{O}_{X}\right)$ be a locally ringed space. A sheaf of $\mathcal{O}_{X}$-modules $\mathcal{F}$ is called quasi-coherent if for every point $x \in X$ there exists an open neighbourhood $x \in U \subset X$ such that $\left.\mathcal{F}\right|_{U}$ is isomorphic to the cokernel of a map

$$
\left.\left.\bigoplus_{j \in J} \mathcal{O}_{X}\right|_{U} \rightarrow \bigoplus_{i \in I} \mathcal{O}_{X}\right|_{U}
$$

Remark 6.4 If $\left(X, \mathcal{O}_{X}\right)$ is a complex manifold, then we need the category of Fréchet quasi-coherent sheaves, which is a variation of the category of quasi-coherent sheaves, see [8, Section 4.3] for more details.

The natural inclusion $i: \mathrm{Q} \operatorname{coh}(X) \rightarrow \operatorname{Sh}(X)$ induces a natural functor

$$
\widetilde{i}: \mathrm{D}(\mathrm{Q} \operatorname{coh}(X)) \rightarrow \mathrm{D}_{\mathrm{Q} c o h}(X),
$$

where $\mathrm{D}_{\mathrm{Qcoh}}(X)$ is the derived category of complexes of $\mathcal{O}_{X}$-modules with quasicoherent cohomologies. However, the functor $\widetilde{i}$ is not necessarily essentially surjective nor fully faithful. The same is true when we restrict to certain subcategories such as perfect complexes or coherent complexes.

Since $\tilde{i}: \mathrm{D}(\mathrm{Q} \operatorname{coh}(X)) \rightarrow \mathrm{D}_{\mathrm{Q} c o h}(X)$ is not an equivalence in general, we need to impose some condition on the locally ringed space $\left(X, \mathcal{O}_{X}\right)$ for our purpose, see Definitions 3.22 and 5.16. It is important to verify for which $X$ this condition holds. In fact we have the following result.

Proposition 6.5 ([17, Proposition B.16], [2, Exposé II, 3.5]) Let X be either a quasicompact and semi-separated scheme, or else a Noetherian scheme. Then the functor $\widetilde{i}: \mathrm{D}^{+}(\mathrm{Qcoh}(X)) \rightarrow \mathrm{D}_{\mathrm{Qcoh}}^{+}(X)$ is an equivalence, where $\mathrm{D}^{+}(\mathrm{Qcoh}(X))$ is the derived category of complexes of quasi-coherent modules with bounded below cohomologies, and $\mathrm{D}_{\mathrm{Qcoh}}^{+}(X)$ is the derived category of complexes of $\mathcal{O}_{X}$-modules with bounded below and quasi-coherent cohomologies.

Corollary 6.6 Any quasi-compact and semi-separated or Noetherian scheme satisfies the perfect-equivalent condition.

Proof On a quasi-compact scheme, any perfect complex has bounded below cohomology, hence by Proposition 6.5 any quasi-compact scheme satisfies the perfectequivalent condition. 
However, a bounded above coherent complex is not necessarily bounded below hence we can no longer use Proposition 6.5. Nevertheless, we have the same result under additional conditions.

Proposition 6.7 ([17, B.17]) Let X be either a Noetherian scheme of finite Krull dimension or a semi-separated scheme with underlying space a Noetherian space of finite Krull dimension. Then the functor $\widetilde{i}: \mathrm{D}(\mathrm{Q} \operatorname{coh}(X)) \rightarrow \mathrm{D}_{\mathrm{Qcoh}}(X)$ is an equivalence.

Hence we immediately have

Corollary 6.8 Any Noetherian scheme of finite Krull dimension or a semi-separated scheme with underlying space a Noetherian space of finite Krull dimension satisfies the coherent-equivalent condition.

\section{Appendix 2: Good covers of locally ringed spaces}

We discuss good covers of locally ringed spaces in this appendix. Recall Definitions 3.16, 3.18, 5.11, and 5.13. The definition of good cover is not too restrictive since we have the following examples of ringed spaces with good covers:

- $\left(X, \mathcal{O}_{X}\right)$ is a separated scheme, then any affine cover is both p-good and c-good. In fact, on a separated scheme the intersection of two affine open subsets is still affine hence the second condition of Definition 3.16 is obviously satisfied and the first condition is proved in [17, Proposition 2.3.1].

- $\left(X, \mathcal{O}_{X}\right)$ is a complex manifold with $\mathcal{O}_{X}$ the sheaf of holomorphic functions. In this case a Stein cover is both p-good and c-good. Actually on complex manifolds we should use the definition of Fréchet quasi-coherent sheaves, which is a variation of ordinary quasi-coherent sheaves, see [8, Section 4]. A Stein manifold satisfies the second condition of Definition 3.16 by [8, Proposition 4.3.3], and the first condition can be proved in the same way as the argument in [17, Section 2].

- $\left(X, \mathcal{O}_{X}\right)$ is a paracompact topological space with soft structure sheaf $\mathcal{O}_{X}$. Then any contractible open cover is both p-good and c-good.

Acknowledgments The author would like to thank Jonathan Block for introducing the author to this topic and for numerous discussions. He also wants to thank Valery Lunts, Olaf Schnürer, Julian Holstein, Ian Shipman and Shilin Yu for very helpful conversations and comments and thank Leo Alonso, Adeel Khan, Daniel Miller, Denis Nardin, and Jeremy Rickard for answering questions related to this work. The author also want to thank the referee for his/her very careful work.

\section{References}

1. Ben-Bassat, O., Block, J.: Milnor descent for cohesive dg-categories. J. K-Theory 12(3), 433-459 (2013)

2. Berthelot, P., Grothendieck, A., Illusie, L. (eds.): Théorie des Intersections et Théorème de RiemannRoch (SGA 6)—Séminaire de Géométrie Algébrique du Bois Marie 1966-1967. Lecture Notes in Mathematics, vol. 225. Springer, Berlin (1971) 
3. Block, J.: Duality and equivalence of module categories in noncommutative geometry. In: Kotiuga, P.R. (ed.) A Celebration of the Mathematical Legacy of Raoul Bott. CRM Proceedings \& Lecture Notes, vol. 50, pp. 311-339. American Mathemetical Society, Providence (2010)

4. Block, J., Holstein, J.V.S., Wei, Z.: Explicit homotopy limits of dg-categories and twisted complexes (2015). arXiv:1511.08659

5. Block, J., Smith, A.M.: The higher Riemann-Hilbert correspondence. Adv. Math. 252, 382-405 (2014)

6. Bökstedt, M., Neeman, A.: Homotopy limits in triangulated categories. Compositio Math. 86(2), 209234 (1993)

7. Bondal, A.I., Kapranov, M.M.: Framed triangulated categories. Sb. Math. 70(1), 93-107 (1991)

8. Eschmeier, J., Putinar, M.: Spectral Decompositions and Analytic Sheaves. London Mathematical Society Monographs. New Series, vol. 10. The Clarendon Press, New York (1996)

9. Gillet, H.: The $K$-theory of twisted complexes. In: Bloch, S.J., et al. (eds.) Applications of Algebraic $K$-theory to Algebraic Geometry and Number Theory, I, Contemporary Mathematics, vol. 55, pp. 159-191. American Mathematical Society, Providence (1986)

10. Holstein, J.V.S.: Properness and simplicial resolutions in the model category dgCat. Homology Homotopy Appl. 16(2), 263-273 (2014)

11. Hovey, M.: Model category structures on chain complexes of sheaves. Trans. Amer. Math. Soc. 353(6), 2441-2457 (2001)

12. Lunts, V.A., Schnürer, O.M.: New enhancements of derived categories of coherent sheaves and applications. J. Algebra 446, 203-274 (2016)

13. Lurie, J.: Higher Algebra (2014). http://www.math.harvard.edu/ lurie/papers/higheralgebra.pdf

14. O'Brian, N.R., Toledo, D., Tong, Y.L.L.: The trace map and characteristic classes for coherent sheaves. Amer. J. Math. 103(2), 225-252 (1981)

15. O'Brian, N.R., Toledo, D., Tong, Y.L.L.: A Grothendieck-Riemann-Roch formula for maps of complex manifolds. Math. Ann. 271(4), 493-526 (1985)

16. Tabuada, G.: Homotopy theory of dg categories via localizing pairs and Drinfeld's dg quotient. Homology Homotopy Appl. 12(1), 187-219 (2010)

17. Thomason, R.W., Trobaugh, T.: Higher algebraic $K$-theory of schemes and of derived categories. In: Cartier, P., et al. (eds.) The Grothendieck Festschrift, III, Progress in Mathematics, vol. 88, pp. 247-435. Birkhäuser, Boston (1990)

18. Toën, B.: Lectures on dg-categories. In: Baum, P.F. (ed.) Topics in Algebraic and Topological K-Theory. Lecture Notes in Mathematics, vol. 2008, pp. 243-302. Springer, Berlin (2011)

19. Toledo, D., Tong, Y.L.L.: Duality and intersection theory in complex manifolds. I. Math. Ann. 237(1), 41-77 (1978)

20. Stacks Project (2015). http://stacks.math.columbia.edu 